\title{
Genome-scale screening of deubiquitinase subfamily identifies USP3 as a stabilizer of Cdc25A regulating cell cycle in cancer
}

\author{
Soumyadip Das ${ }^{1} \cdot$ Arun Pandian Chandrasekaran ${ }^{1} \cdot$ Bharathi Suresh $^{2} \cdot$ Saba Haq $^{3} \cdot$ Jae-Hyeok Kang $^{3} \cdot \mathrm{Su}^{-J a e}$ Lee $^{3}$. \\ Jaewon $\mathrm{Kim}^{4}$ - Jaesang $\mathrm{Kim}^{5}$. Sanghyuk Lee ${ }^{4,5} \cdot$ Hyongbum Henry Kim ${ }^{2,6,7,8,9} \cdot$ Kye-Seong Kim ${ }^{1,10}$. \\ Suresh Ramakrishna ${ }^{1,10}$
}

Received: 5 November 2019 / Accepted: 4 May 2020 / Published online: 15 May 2020

(c) The Author(s), under exclusive licence to ADMC Associazione Differenziamento e Morte Cellulare 2020

\begin{abstract}
Conventional screening methods for deubiquitinating enzymes (DUBs) have important limitations. A loss-of-function study based on the knockout of DUB genes in mammalian cells can provide an excellent model for exploring DUB function. Here, we used CRISPR-Cas9 to perform genome-scale knockout of the entire set of genes encoding ubiquitin-specific proteases (USPs), a DUB subfamily, and then systematically screened for DUBs that stabilize the Cdc25A oncoprotein. USP3 was identified as a deubiquitinase of Cdc25A. USP3 depletion reduces the Cdc25A protein level, resulting in a significant delay in cell-cycle progression, and reduces the growth of cervical tumor xenografts in nude mice. Clinically, USP3 expression is positively correlated with Cdc25A protein expression and the poorest survival in breast cancer. We envision that our DUB knockout library kit will facilitate genome-scale screening of functional DUBs for target proteins of interest in a wide range of biomedical fields.
\end{abstract}

These authors contributed equally: Soumyadip Das, Arun Pandian Chandrasekaran, Bharathi Suresh

\section{Edited by S. Kumar}

Supplementary information The online version of this article (https:// doi.org/10.1038/s41418-020-0557-5) contains supplementary material, which is available to authorized users.

\footnotetext{
$\triangle$ Hyongbum Henry Kim

hkim1@yuhs.ac

$\triangle$ Kye-Seong Kim

ks66kim@hanyang.ac.kr

$\triangle$ Suresh Ramakrishna

suri28@ hanyang.ac.kr

1 Graduate School of Biomedical Science and Engineering, Hanyang University, Seoul, Republic of Korea

2 Department of Pharmacology, Yonsei University College of Medicine, Seoul, Republic of Korea

3 Department of Life Science, College of Natural Sciences, Hanyang University, Seoul, Republic of Korea
}

\section{Introduction}

The cell cycle is a tightly regulated process controlled by a signaling network that ensures normal cell division [1,2]. The cell division cycle 25 (Cdc25) proteins are dualspecificity phosphatases that activate cyclin-dependent kinase (CDK) complexes to ensure the proper progression of the cell division cycle [3]. Cdc25A, a Cdc25 phosphatase family member, plays a key role in regulating both the G1/S and G2/M progressions by activating CDKs through dephosphorylation at the threonine and tyrosine residues [4].

4 Department of Bio-Information Science, Ewha Womans University, Seoul, Republic of Korea

5 Department of Life Science, Ewha Womans University, Seoul, Republic of Korea

6 Brain Korea 21 Plus Project for Medical Sciences, Yonsei University College of Medicine, Seoul, Republic of Korea

7 Severance Biomedical Science Institute, Yonsei University College of Medicine, Seoul, Republic of Korea

8 Center for Nanomedicine, Institute for Basic Science (IBS), Seoul, Republic of Korea

9 Yonsei-IBS Institute, Yonsei University, Seoul, Republic of Korea

10 College of Medicine, Hanyang University, Seoul, Republic of Korea 
Cdc25A switches from a steady state to a labile one during mitosis and interphase in a process regulated by its posttranslational modification [3]. In response to DNA damage, cells undergo rapid $\mathrm{G} 1$ arrest due to the proteasomal degradation of Cdc25A. Notably, overexpression of Cdc $25 \mathrm{~A}$ in cancer has frequently been associated with genetic instability and checkpoint abrogation, contributing to malignancy and poor prognosis $[5,6]$.

Posttranslational modifications play a critical role in the maintenance of Cdc25A stability. Cdc25A protein levels vary in a cell-cycle-dependent manner, with predominant expression in G1 and stabilization in mitosis through CDK1-cyclin B-mediated phosphorylation. Cdc25A is then subjected to anaphase promoting complex/cyclosome (APC/C)-dependent ubiquitination [7]. During the S and G2 phases, Cdc25A undergoes proteasome-mediated degradation by the $\mathrm{SCF}^{\beta \mathrm{TRCP}}$ (Skp1/Cullin/F-box) ubiquitin ligase complex [7]. Although much attention has been given to the ubiquitination system in the maintenance of Cdc25A stability, the reversal of its ubiquitination by deubiquitinating enzymes (DUBs) plays an equally critical role in the progression of the cell division cycle [8].

In this study, we performed a CRISPR-Cas9 (clustered regularly interspaced short palindromic repeats/CRISPRassociated-9)-based screening of ubiquitin-specific proteases (USPs) to identify candidates that might regulate $\mathrm{Cdc} 25 \mathrm{~A}$ protein turnover by reversing its ubiquitination, thereby protecting it from degradation. We generated a library of 50 individual knockout cell lines targeting the genes of each USP family member. The cell lysates from these USP knockout cell lines were used in western blot analyses to screen for DUB candidates that regulate Cdc25A protein stabilization. Our approach can provide new insights into the molecular mechanisms by which DUBs regulate their target proteins in diverse cellular processes.

\section{Materials and methods}

\section{Plasmids, antibodies, and reagents}

HA-Cdc25A, Flag-APC/Cdh1, and Flag- $\beta$ TrCP were kindly given to us by Dr Zhao-Qi Wang. The HA-Cdc25A was further subcloned into the pcDNA 3.1 6XMyc-vector. pAdDsRed-IRES-EGFP-Cdc25A was a kind gift from Dr Tiebang Kang. Flag-USP3 (\#22582), HA-ubiquitin (\#18712), GFPH2B (\#11680), and Cas9-2A-GFP (\#44719) were purchased from Addgene. To generate the catalytic mutant of USP3, the active cysteine residue at position 168 was replaced with serine, producing USP3C168S (USP3CS) by site-directed mutagenesis. Cas9-2A-mRFP-2A-PAC was purchased from
Toolgen (Seoul, South Korea). The antibodies used for coimmunoprecipitation, immunofluorescence, flow cytometry, and western blotting were anti-Cdc25A (Santa Cruz Biotechnology sc-7389, Abcam ab989, and Proteintech 55031-1AP at a dilution of 1:1000 for western blots, 1:500 for immunofluorescence, and 1:100 for immunohistochemistry), anti-USP3 (Abcam ab101473, Genetex GTX128238, and Novus NB100-77284 at a dilution of 1:1000 for western blots, 1:500 for immunofluorescence, and 1:100 for immunohistochemistry), anti-Cdc25B (Santa Cruz Biotechnology, sc-326, 1:1000), anti-Cdc25C (Santa Cruz Biotechnology, sc13138, 1:1000), anti-cyclin B (Santa Cruz Biotechnology, sc-166152, 1:1000), anti-cyclin B1 (Cell Signaling Technology, 12231, 1:1000), anti-phospho-cdc2 (Tyr15) (Cell Signaling Technology, 4539), anti-CDK1 (Santa Cruz Biotechnology, sc-53219, 1:1000), anti-phospho-histone H3 (Ser10) (Cell Signaling Technology, 3377, at a dilution of 1:500 for immunofluorescence), anti-BrdU (Biolegend, 339811, at a dilution of 1:500 for flow cytometry), anti-Flag (anti-DDDDK-tag) (MBL International, M185-3L, 1:1000), anti-Myc (Santa Cruz Biotechnology, sc-40, 1:1000), anti-ubiquitin (Santa Cruz Biotechnology, sc-8017, 1:1000), anti-HA (Santa Cruz Biotechnology, sc-7392, 1:1000), antiDsRed (Santa Cruz Biotechnology, sc-390909, 1:1000), anti-GFP (Santa Cruz Biotechnology, sc-9996, 1:1000), anticleaved-PARP (Cell Signaling Technology, 5625S, 1:100 for immunofluorescence), and TUBE 2 (Life Sensors, UM402).

\section{Cell culture and treatments}

Human embryonic kidney (HEK293), cervical cancer (HeLa), osteosarcoma (U2OS), colorectal adenocarcinoma (Caco2), prostate cancer (PC3), breast cancer (MCF7), and colorectal adenocarcinoma (SW620) cells (ATCC, VA, USA) were cultured in DMEM (GIBCO BRL, Rockville, MD, USA) supplemented with $10 \%$ fetal bovine serum (GIBCO) and $1 \%$ penicillin and streptomycin (GIBCO BRL, Rockville, MD, USA) at $37^{\circ} \mathrm{C}$ in a humidified atmosphere with $5 \% \mathrm{CO}_{2}$. Human dental pulp stem cells (hDPSCs) were cultured in $\alpha$ modified Eagle medium (Gibco). PR-619 was purchased from Abcam (ab144641). Cycloheximide (CHX) was purchased from Sigma-Aldrich. Cells were treated with nocodazole (Sigma-Aldrich), bromodeoxyuridine (Biolegend, CA, USA), etoposide (Sigma-Aldrich), AZ20 (Sigma-Aldrich), and caffeine (Sigma-Aldrich) at the indicated concentrations.

\section{Cas9 and sgRNA constructs}

To screen the single guide RNAs (sgRNAs), we used a plasmid encoding Cas9-2A-mRFP-2A-PAC (puromycin $\mathrm{N}$-acetyl-transferase and puromycin resistance gene) and a plasmid encoding the sgRNAs; both constructs were 
purchased from Toolgen (Seoul, South Korea). The sgRNA target sequences were designed using bioinformatics tools (www.broadinstitute.org) and cloned into the vectors as previously described [9]. Briefly, oligonucleotides containing each target sequence were synthesized (Bioneer, Seoul, South Korea), and T4 polynucleotide kinase was used to add terminal phosphates to the annealed oligonucleotides (Biorad, CA, USA). As a mock control, non-targeted sgRNAs (scrambled sgRNA sequences) were designed. The vector was digested with BsaI restriction enzyme and ligated with the annealed oligonucleotides. The oligonucleotide sequences are listed in Table S1.

\section{T7E1 assay}

The T7 endonuclease I (T7E1) assay was performed as previously described $[10,11]$. Isolation of genomic DNA was performed using DNeasy Blood \& Tissue kits (Qiagen, Hilden, Germany) according to the manufacturer's instructions. The region of DNA containing the nuclease target site was PCR-amplified using hemi-nested or nested primers. Amplicons were denatured by heating and annealed to form heteroduplex DNA, which was then treated with 5 units of T7E1 (New England Biolabs, MA, USA) for $15-20 \mathrm{~min}$ at $37^{\circ} \mathrm{C}$, followed by $2 \%$ agarose gel electrophoresis. Mutation frequencies were calculated based on band intensity by ImageJ software using the following equation: mutation frequency $(\%)=100 \times$

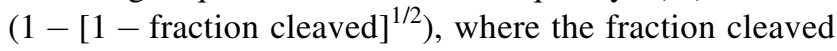
was the total relative density of the cleavage bands divided by the sum of the relative density of the cleavage and uncut bands. The oligonucleotide sequences used to get the PCR amplicons for the T7E1 assay are listed in Table S2. The amplicon size of each USP gene and the expected cleavage sizes after the T7E1 assay are summarized in Table S3.

\section{Generation of DUB knockout single cell-derived clones}

To generate the DUB knockout library, HEK293 cells were co-transfected with the plasmid encoding Cas9 and sgRNAs targeting USP genes at a 1:2 weight ratio using Lipofectamine 2000 (Invitrogen, Carlsbad, CA, USA). To generate mock clones, HEK293 cells were co-transfected with the plasmid encoding Cas9 and non-targeted sgRNAs (scrambled sgRNAs) at a 1:2 weight ratio and further subjected to clonal selection. One day after transfection, the transfected cells were selected with puromycin $(1 \mu \mathrm{g} / \mathrm{mL})$ for $48 \mathrm{~h}$, and then a small portion of the cells was harvested for the T7E1 assay to estimate the sgRNA efficiency. The cells transfected with sgRNA and showing higher than average indel frequency were chosen for generating the single cell-derived clones. The cells were trypsinized, resuspended in DMEM, and seeded into 96well plates at an average density of 0.25 cells/well. Sixteen days after cell seeding, each well was microscopically evaluated, and round, single cell-derived colonies were selected [12]. Each selected colony was individually trypsinized and replated into a 24-well plate. Seven days after subculture, the single cell-derived knockout clones were harvested for their genomic DNA and subjected to T7E1 analysis. T7E1-positive clones were expanded and stored in liquid nitrogen. Gene disruption was confirmed by sequencing the region including the target sequence, as previously described [12]. Briefly, PCR amplicons that included the CRISPR-Cas9-target sites were cloned into the T-Blunt vector. The cloned plasmids were sequenced with the same primers used for PCR amplification, as summarized in Table S2.

\section{Transfection and transduction}

For plasmid transfection, HeLa, HEK293, and U2OS cells were transfected with Lipofectamine 2000 (Invitrogen, Carlsbad, CA, USA) according to the manufacturer's protocol. Short hairpin RNA (shRNA) lentiviral particles against USP3 were transduced according to the manufacturer's protocol. To generate USP3 knockout in HeLa cells, sgRNA2 targeting USP3 was transfected into cells along with Cas9-endonuclease. Similarly, to generate Cdc25A knockout in HeLa cells, sgRNA2 targeting Cdc25A was transfected along with Cas9-endonuclease. The transfected cells were puromycin selected $(1.5 \mu \mathrm{g} / \mathrm{mL})$ and reseeded into 96-well plates to establish single cellderived colonies. After 12 days, single round colonies were marked and expanded for screening by the T7E1 assay.

\section{Immunoprecipitation}

Forty-eight hours after transfection, the cells were harvested and lysed in buffer B (50 mM Tris pH 7.6, $150 \mathrm{mM} \mathrm{NaCl}$, $1 \mathrm{mM}$ EDTA, $1 \%$ Triton X-100, $1 \mathrm{mM}$ PMSF) for $20 \mathrm{~min}$. Immunoprecipitation was carried out by incubating the precleared lysates with the indicated antibodies at $4{ }^{\circ} \mathrm{C}$ overnight, followed by $20 \mu \mathrm{L}$ of protein A/G Sepharose beads (Santa Cruz Biotech, TX, USA) at $4{ }^{\circ} \mathrm{C}$ for $2 \mathrm{~h}$. The beads were washed with lysis buffer and eluted with SDS sample buffer. Co-immunoprecipitated proteins were examined by western blot analysis and blotted with the indicated primary antibodies. Rabbit IgG TrueBlot secondary antibody (Rockland Antibodies, Cat 18-8816-31) was used to prevent interference from heavy and light immunoglobulin chains in the binding assays. 


\section{Reporter assay}

HEK293 cells were co-transfected with a reporter plasmid encoding DsRed/EGFP-Cdc25A along with the constructs indicated in Fig. 3c, f (1:1 ratio). After 48 h, the live cells were harvested, resuspended in phosphate-buffered saline (PBS) with $10 \%$ fetal bovine serum and filtered using a $40 \mu \mathrm{m}$ cell strainer (BD Falcon, Franklin Lakes, NJ, USA). The filtered cells were subjected to flow-cytometry analysis on a FACS flow cytometer (BD FACSCANTO II, BD Biosciences).

\section{Immunofluorescence}

HeLa cells were transfected with the constructs indicated in Figs. $3 \mathrm{i}$ and $5 \mathrm{~b}$, e, and $5 \mathrm{~g}$ were grown on glass coverslips for $48 \mathrm{~h}$, and then fixed in $4 \%$ paraformaldehyde in PBS for $10 \mathrm{~min}$ at room temperature. After washing with PBS, the cells were permeabilized in PBS with $0.1 \%$ Triton for $5 \mathrm{~min}$, washed extensively in PBS, and incubated with specific primary antibodies diluted in bovine serum albumin overnight. On the following day, the cells were incubated with appropriate conjugated secondary antibodies for $1 \mathrm{~h}$. They were then washed several times in PBS and mounted on glass slides. The cells were visualized, and images were captured using a Leica fluorescence microscope (Leica, DM5000 B; Leica CTR 5000; Germany).

\section{Real-time PCR}

Total RNA was isolated using Trizol reagent (Favorgen, Kaohsiung, Taiwan). RNA pellets were suspended in $30 \mu \mathrm{L}$ nuclease-free water, after which the RNA concentration was determined. Total mRNA was reverse transcribed into cDNA using the SuperScript III FirstStrand Synthesis System (Life Technologies) with an oligo dT primer. Quantitative PCR was performed using Fast SYBR Green master mix (Life Technologies) and a StepOnePlus Real-Time PCR System (Life Technologies, USA) with GAPDH-targeting primers (5'-CATGTTCGT CATGGGTGTGAACCA-3', 5'-AGTGATGGCATGGAC TGTGGTCAT-3 $\left.{ }^{\prime}\right)$ and Cdc25A-targeting primers $\left(5^{\prime}\right.$ CAGCTTCCACACCAGTCTCT-3' and 5'-TTGACTGC CGATACCCATAT-3').

\section{TUBEs ubiquitination assay}

The ubiquitination of Cdc25A was validated in HeLa cells using the Tandem Ubiquitin Binding Entities (TUBEs) ubiquitination assay. The required number of cells was seeded and incubated overnight. Then, IL-1 $\beta$ ( $5 \mathrm{ng} / \mathrm{mL}$, Sigma-Aldrich) was added for the time intervals ( 0 and $10 \mathrm{~min}$ ). Cells were harvested and lysed, and the pUb chains were captured from the cell lysate using TUBEs. Then, the samples were subjected to western blotting with the indicated antibodies.

\section{Deubiquitination assay}

The DUB activity of USP3 and its effect on Cdc25A were examined in HEK293 and HeLa cells transfected with the constructs indicated in Fig. 4a-c. After $48 \mathrm{~h}$, the cells were treated (or not) with MG132 $(5 \mu \mathrm{M} / \mathrm{mL})$ for $4 \mathrm{~h}$ and harvested, followed by immunoprecipitation and western blotting with the indicated antibodies.

\section{Cell cycle and apoptosis assays}

For the cell-cycle experiments, transfected HeLa cells were washed twice with PBS, fixed in ice-cold $70 \%$ ethanol, and resuspended in PBS containing propidium iodide (PI) $(50 \mu \mathrm{g} / \mathrm{mL}$; Sigma $)$ and RNase A $(200 \mu \mathrm{g} / \mathrm{mL}$, New England Biolabs, MA, USA). Analyses were performed with a FACS flow cytometer (BD FACSCANTO II, BD Biosciences) and Kaluza Beckman-Coulter software. To block cells at the M-phase, transfected HeLa cells were treated with nocodazole for $16 \mathrm{~h}$, followed by mitotic release with fresh medium. To estimate the change in mitotic progression, asynchronized and nocodazole-released cells were subjected to PI staining to estimate their DNA content. To identify cells in the $\mathrm{S}$ phase, transfected HeLa cells were treated with bromodeoxyuridine (BrdU, $100 \mu \mathrm{g} / \mathrm{mL}$, Biolegend) for $1 \mathrm{~h}$ and harvested in PBS. The harvested cells were fixed in $70 \%$ ethanol, pelleted, resuspended in $\mathrm{HCl}$, treated with sodium tetraborate, and then stained with AntiBrdU (1:250, Biolegend, CA, USA) according to the manufacturer's protocol. HeLa cells were transfected with the constructs indicated in Fig. 5c, followed by etoposide treatment for $12 \mathrm{~h}$, and dead cells were identified by their sub-diploid (sub-G1) DNA content. Apoptotic populations were quantified using a PE annexin-V/7-AAD staining kit (BD Biosciences) according to the manufacturer's protocol. In brief, HeLa cells were transfected with the constructs indicated in Fig. 5f and harvested after $48 \mathrm{~h}$. Then, the cells were washed with ice-cold PBS containing $10 \%$ fetal bovine serum. The required cells were counted, and $5 \mu \mathrm{L}$ of annexin- $\mathrm{V}$ and 7-AAD was added. Then, the cells were incubated in the dark for $15 \mathrm{~min}$. Stained cells were suspended with binding buffer, and flow cytometry was performed within $1 \mathrm{~h}$.

\section{Time-lapse microscopy}

Time-lapse live cell imaging was performed in a $\mathrm{CO}_{2}$ chamber at $37^{\circ} \mathrm{C}$ (Applied Precision) using the $40 \times$, 1.35NA, $0.10 \mathrm{~mm}$, WD objective lens on a microscope (Delta Vision Core; GE Healthcare) equipped with a 
charge-coupled device camera (photometrics). U2OS cells transfected with Cas9-endonuclease and sgRNA targeting USP3 were FACS-sorted for Cas9-positive cells, and the sorted cells were then expanded to generate enriched USP3 transient knockout cells. Those cells were transfected with histone H2B-GFP (Addgene, \#11680) in a glass-bottom multi-well chamber (eight-well chamber, Lab-Tek II chambered cover glass, Thermo Fisher). Time-lapse images were taken at $1 \mathrm{~min}$ intervals and maximally projected. Data were obtained from three independent experiments.

\section{Clonogenic survival assay}

HeLa cells transfected with the constructs indicated in Supplementary Fig. S11a were incubated with etoposide for 14 days. After 14 days, the surviving colonies were counted under light microscopy by staining with crystal violet. All experiments were performed in triplicate and are graphically represented.

\section{Cell proliferation assay}

Mock, USP3KO, and Cdc25AKO HeLa cells were transfected with the constructs indicated in Fig. 6a, b and seeded in 96-well plates with $200 \mu \mathrm{L}$ of culture medium. Next, $10 \mu \mathrm{L}$ of cell counting kit-8 assay reagent (CCK-8; Dojindo Molecular Technologies, Rockville, MD) was added to each well, and the cells were cultured for $2 \mathrm{~h}$. Absorbance was measured using a spectrophotometer (Bio-Rad Laboratories, Inc., Korea) at $450 \mathrm{~nm}$. In experiments with ATR inhibitors, AZ20 $(10 \mu \mathrm{M})$ or caffeine $(150 \mathrm{mM})$ was used to treat cells for $48 \mathrm{~h}$. Data were obtained from three independent experiments.

\section{Soft agar assay}

Mock, USP3KO, and Cdc25AKO HeLa cells were transfected with the constructs indicated in Fig. 6e. First, $1 \mathrm{~mL}$ of $0.75 \%$ agarose gel with $1 \times$ DMEM complete (equal ratio) was placed into six-well plates and held for $45 \mathrm{~min}$ at room temperature. HeLa cells $\left(1 \times 10^{4}\right)$ were suspended in $500 \mu \mathrm{L}$ of DMEM containing $500 \mu \mathrm{L}$ of $0.75 \%$ agarose and inoculated onto the surface of the solidified gel. Cells were cultured for 15 days at $37^{\circ} \mathrm{C}$ in a humidified atmosphere containing 5\% $\mathrm{CO}_{2}$. The anchorage-independent colony numbers were counted under light microscopy after staining with crystal violet. Data were obtained from three independent experiments.

\section{Wound healing assay}

Migration activity was analyzed by the wound healing assay. Mock, USP3KO, and Cdc25AKO HeLa cells were transfected with the constructs indicated in Fig. 6d and cultured to near $90 \%$ confluency. Scratches were made in the monolayers with a sterile pipette tip in a definite array. The wounded cell layer was washed with PBS and incubated with serum-free medium. Wound closure or the area of the wound was compared at 0 and $24 \mathrm{~h}$ using a fluorescence microscope (IX71, Olympus, Tokyo, Japan) and ImageJ software. The percentage of wound healing was calculated as previously described [13]. Data were obtained from three independent experiments.

\section{Matrigel invasion assay}

Cell invasion was assessed using $0.8 \mu \mathrm{m}$ Transwell chambers (Falcon) coated with Matrigel (Corning, USA) according to the manufacturer's protocol. Briefly, $2.5 \times 10^{4}$ cells suspended in $500 \mu \mathrm{L}$ of serum-free DMEM were placed in 24-well chambers. Next, $750 \mu \mathrm{L}$ of complete medium was added, and the cells were incubated in a $5 \%$ $\mathrm{CO}_{2}$ atmosphere at $37^{\circ} \mathrm{C}$. After $24 \mathrm{~h}$, the cells on the upper surface of the insert were scraped off, and the cells on the lower surface were fixed with ice-cold methanol and stained with crystal violet. The average number of cells was counted using light microscopy. Data were obtained from three independent experiments.

\section{Xenograft tumor experiment}

Male NOD scid $\gamma$ (NSG) mice (6 weeks old) were used for the animal experiments. The animal study was approved by the IACUC. All mice were housed in standard conditions with a $12 \mathrm{~h}$ light/dark cycle and access to food and water. To generate a stable Cdc25A-overexpressing USP3KO cell line, we transfected USP3KO cells with the HA-Cdc25A plasmid and selected with an antibiotic (G418) for 14 days. HA-Cdc25A expression was confirmed by western blot analysis. Animals were randomized into three groups (five mice per group) and received the following injection, Mock control cells, USP3-depleted HeLa cells, or Cdc25Aoverexpressing USP3KO cells $\left(5 \times 10^{6}\right)$ in PBS: Matrigel (BD Biosciences) (1:1) were subcutaneously injected into the right flanks of mice. The volume of the xenografts was blindly measured by the two co-authors individually every other day for 30 days and estimated using the formula $V=$ $D \times d^{2} / 2$, where $D$ is the long axis, and $d$ is the short axis of the tumor. Upon completion of the measurements, the tumor grafts were harvested. The weight of the harvested tumors was measured, and the tumor tissues from the mouse xenografts were isolated for immunohistochemistry.

\section{Immunohistochemistry}

Xenograft tumor tissue samples were fixed in $4 \%$ paraformaldehyde (Fujifilm Wako Pure Chemical Corporation, 
Osaka, Japan) overnight at $4{ }^{\circ} \mathrm{C}$. Tissues were then dehydrated in $70 \%$ ethanol until they were processed in paraffin. The samples were stained with USP3 (Novus) and Cdc25A (Proteintech) antibodies. Imaging of tumor sections on slides was done on a Leica DM5000 B (Leica, Germany).

Clinical samples, 12 non-neoplastic and 12 invasive ductal carcinomas, were procured from AccMax Array Inc. (ISU Abxis Co., Seoul, South Korea). The formalin-fixed paraffin-embedded tissue specimens were deparaffinized and incubated with anti-USP3 (1:100) or anti-Cdc25A (1:100) according to a protocol previously described [14]. The slides were counterstained with hematoxylin, dehydrated, and mounted. Digital images were captured using a Leica DM5000 B (Leica, Germany).

\section{Expression and survival analysis based on TCGA data}

TCGA expression data sets were downloaded from the UCSC Xena website (https://xenabrowser.net/) as processed data (level 3). Gene expression comparisons between tumor and normal samples were carried out for patients with both tumor and matched normal samples. A paired $t$ test was used to evaluate the $P$ values. A Kaplan-Meier survival analysis was performed for overall survival (OS) using the $\mathrm{R}$ 'survival' package version 2.4. OS was measured as the time from diagnosis to death from any cause. The log rank test was used to evaluate the statistical significance between expression of the gene of interest in the top and bottom tertile groups of patients. A $P$ value $<0.05$ was considered to be statistically significant.

\section{Statistical analysis}

Statistical analyses were conducted in GraphPad Prism 5.0 software (San Diego, USA). All experiments were performed independently at least three times, and the results are presented as means $\pm \mathrm{SD}$. Comparisons between two groups were conducted using the student's $t$ test. One-way ANOVA followed by the Tukey post-hoc test was performed to compare three or more groups. A $P$ value $<0.05$ was considered statistically significant.

\section{Results}

\section{Generation of the DUB knockout library kit using the CRISPR-Cas9 system}

USPs are the largest subfamily of DUBs, comprising about 50 proteins with diverse cellular functions [15-18]. We individually designed two sets of sgRNAs targeting the early exons for the entire set of 50 USP genes. As a mock control, we designed non-targeted, scrambled sgRNAs. We chose HEK293 cells, which express all 50 USP subfamily members [19] (Supplementary Fig. S1) to develop our genome-scale CRISPR/Cas9-mediated DUB knockout library kit targeting the USP subfamily. The HEK293 cells were co-transfected with plasmids encoding Cas 9 and the sgRNAs targeting DUBs. The efficiencies of the sgRNAs were validated as a high indel percentage on the T7E1 assay (Fig. 1a and Supplementary Fig. S2). The sgRNAs with the highest indel percentages were then used to generate single cell-derived DUB knockout (DUBKO) clones. Mock control cells co-transfected with the plasmid encoding Cas9 and non-targeted sgRNAs were also subjected to clonal selection. The transfected cells were diluted and seeded into 96well plates, and the individual single cell-derived clones with disrupted genes were selected using the T7E1 assay (Supplementary Figs. S3 and S4). We successfully generated individual knockout cell lines for all USP genes except USP29, which failed even after several attempts. The transfection of sgRNA targeting USP29 into HEK293 cells caused very low cell viability and poor survival upon longer passages, suggesting that USP29 might behave as a key factor for cell proliferation and survival. The T7E1-positive single cell-derived DUBKO clones with a high indel percentage were subjected to Sanger sequencing to confirm gene disruption. We particularly focused on out-of-frame mutations in the sequencing results (Supplementary Fig. S5). The DUBKO cell lines were individually lysed, and equal concentrations of the lysate aliquots were stored as the DUB screening kit. Notably, all DUBKO lysates can be loaded onto several gels simultaneously to screen for DUB candidates that regulate a protein of interest, demonstrating the usability of our DUBKO cell lysate library as an efficient screening system. We examined potential changes in the expression of our protein of interest $(\mathrm{Cdc} 25 \mathrm{~A})$ in the panel of lysates with the individual loss-of-function DUBs using western blot analyses. A schematic of the DUBKO library design and its application to genome-scale screening for functional DUBs to examine the turnover of target substrates is presented in Fig. 1b.

\section{CRISPR-Cas9 system enables successive genome- scale screening for candidate DUBs regulating the Cdc25A protein}

We used our screening method to identify specific DUBs that stabilize Cdc25A posttranslationally in the hope of identifying new cancer targets. To examine the DUB genes for which loss-of-function confers a reduction in $\mathrm{Cdc} 25 \mathrm{~A}$ protein levels, we examined endogenous $\mathrm{Cdc} 25 \mathrm{~A}$ protein levels in DUBKO cell lysates by western blot analysis (Fig. 2a). In the case of USP29, we used HEK293 cells transiently transfected with Cas9 and sgRNAs targeting the 
a

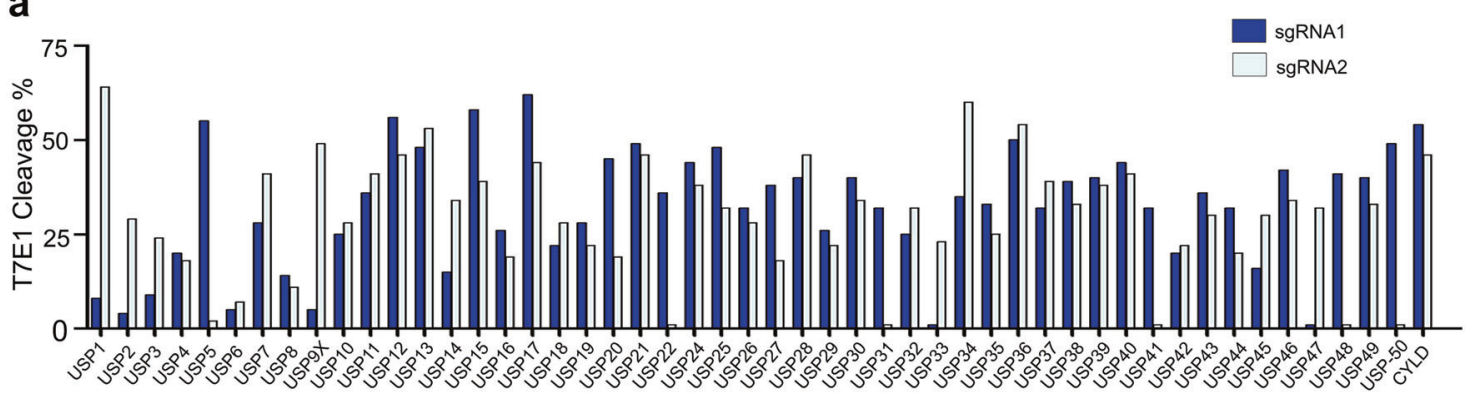

b
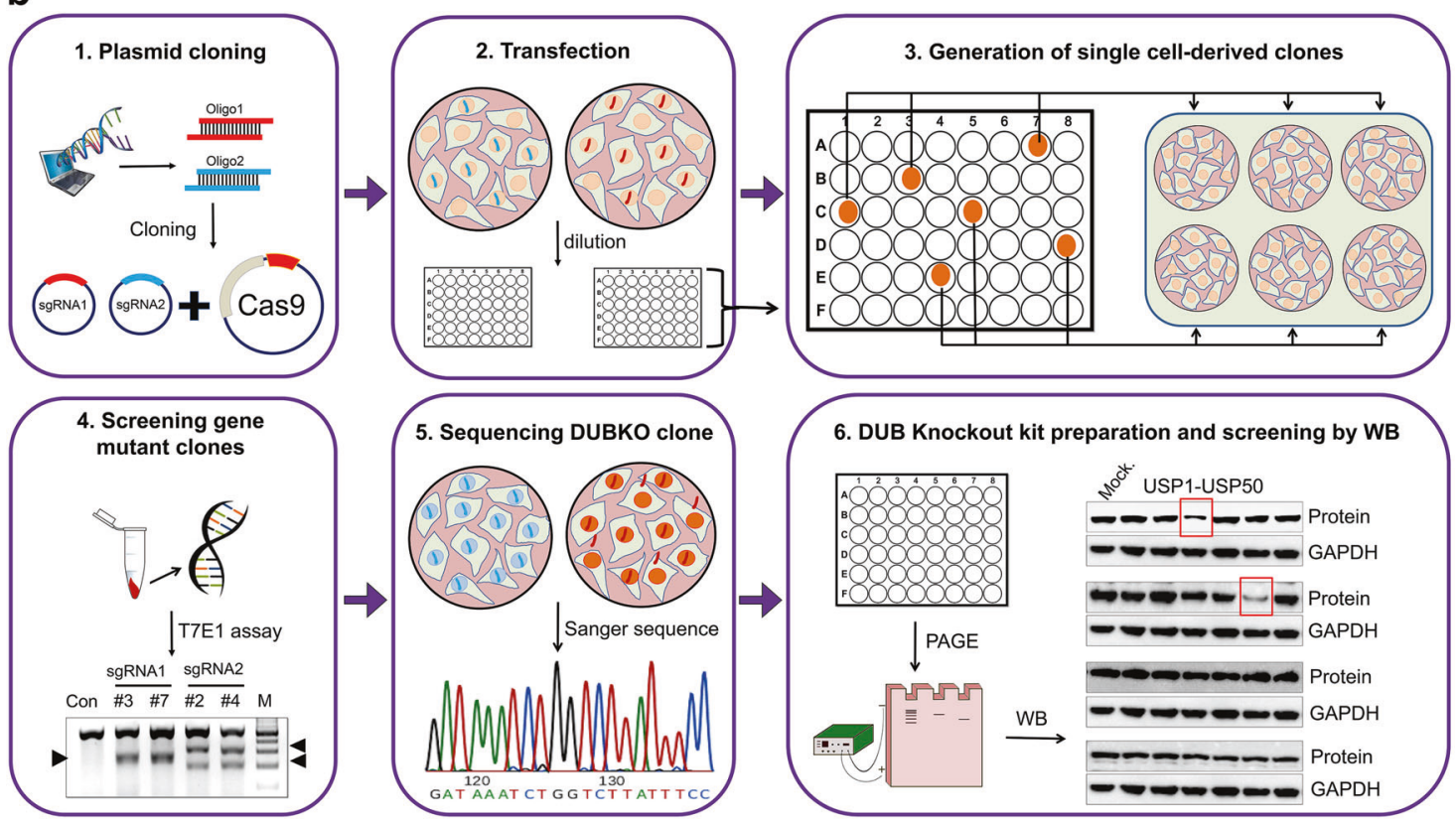

Fig. 1 DUBKO library kit design and application for genome-scale screening for functional DUBs. a The cleavage efficiency of two sets of sgRNAs targeting each USP subfamily protein was determined using the T7E1 assay in HEK293 cells transfected with plasmids encoding Cas9 and the sgRNAs. The cleavage band intensity was measured and graphically represented using ImageJ software. b Scheme illustrating the design of the DUBKO library kit using the CRISPR-Cas9 system. Step 1: Selection of sgRNAs with high cleavage efficiency and the lowest off-target scores using bioinformatics tools and cloning into a plasmid driven by the U6 promoter. Step 2: Transfection and puromycin selection. Cells transfected with the plasmids encoding Cas9 and sgRNAs were diluted in DMEM and seeded into a 96-well plate at an average density of 0.25 cells/well.

USP29 gene, followed by puromycin selection. Compared with the mock controls, the DUBKO cell lysates for USP3 and USP29 showed significantly reduced Cdc25A protein levels, along with DUB3 (USP17) and USP7, both DUBs previously reported to affect Cdc25A [8, 20]. Similar results were obtained when we analyzed the protein level of ectopically expressed Myc-Cdc25A by individually transfecting sgRNAs targeting entire USP subfamily (Supplementary Fig. S6). In contrast, the $\mathrm{Cdc} 25 \mathrm{~B}$ and $\mathrm{Cdc} 25 \mathrm{C}$ isoforms were unaltered (Fig. 2b), confirming that the DUB candidates are specific to $\mathrm{Cdc} 25 \mathrm{~A}$ protein stabilization. We
Step 3: Round, single cell-derived colonies observed through a microscope were transferred to a six-well plate and cultured. Step 4: Screening of the gene-modified DUBKO cell lines by the T7E1 assay. A portion of the cultured cells was harvested for genomic DNA and subjected to T7E1 analysis. Step 5: The sequences of the T7E1positive DUBKO cell lines were confirmed by Sanger sequencing. Step 6: DUBKO kit preparation and screening. Gene disruption confirmed DUBKO cell lines were lysed individually, and equal concentrations of each cell lysate were stored at $-20^{\circ} \mathrm{C}$ as DUBKO kits. The samples from one DUBKO kit were loaded on polyacrylamide gels and screened for a protein of interest by western blot analysis to examine the downregulation of protein levels caused by the loss-offunction of individual DUBs.

also used ectopically expressed Myc-Cdc25A to crossconfirm the effects of the sgRNAs targeting the DUB candidates obtained from the initial screening, and the results show that USP3 and USP29 regulate Cdc25A stability (Fig. 2c).

\section{USP3 as a protein stabilizer of Cdc25A}

We further examined the effects of the DUBs in cancer cells by transiently transfecting human cervical cancer (HeLa) cells with sgRNAs and Cas9. The common DUB inhibitor 
a
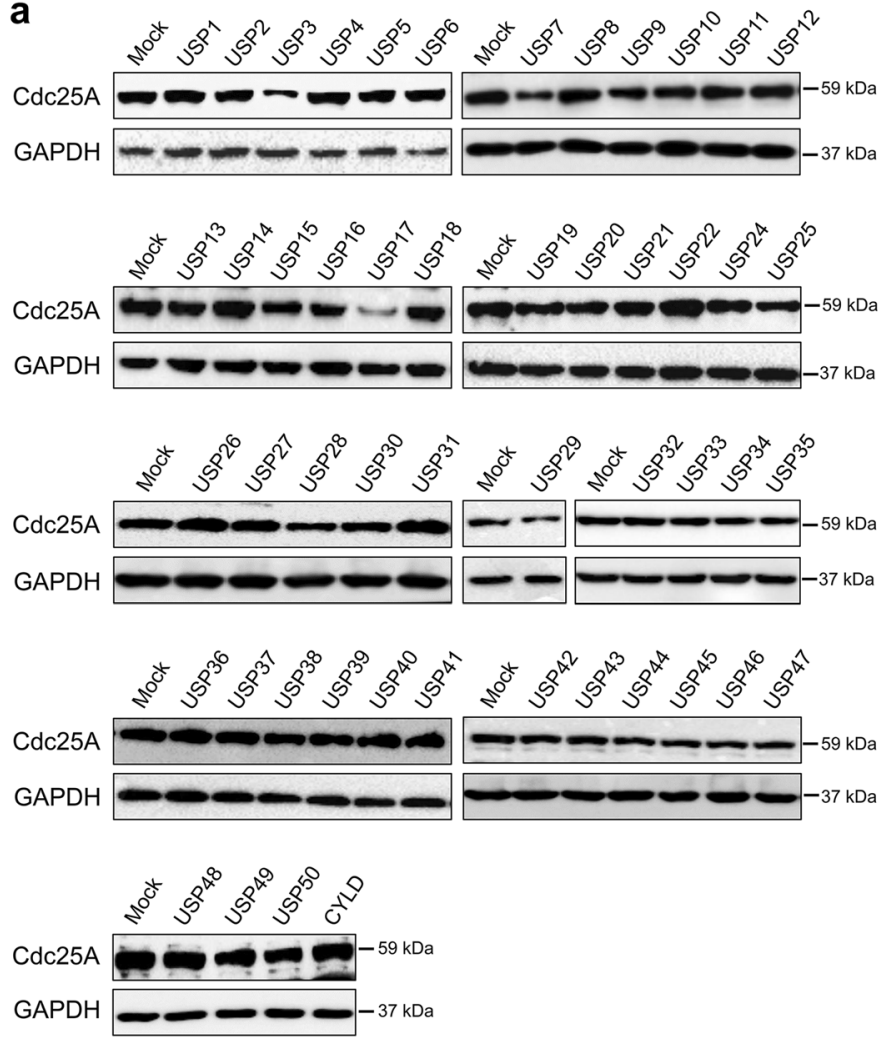

Fig. 2 Screening for functional DUBs that regulate Cdc25A stability using the DUBKO library kit. a Equal protein concentrations of DUBKO HEK293 cell lysates were subjected to western blot analysis to determine endogenous Cdc25A protein levels. The sgRNA targeting USP29 was transiently transfected along with Cas9-endonuclease, and cells were selected by puromycin. GAPDH was used as the loading control. b Top DUB candidates for Cdc25A regulation were analyzed to determine their effects on the endogenous expression of other $\mathrm{Cdc} 25$ isoforms. The graph in the right panel represent the expression of the Cdc25A protein as estimated by ImageJ software. c $6 \times-M y c-C d c 25 \mathrm{~A}$ was co-transfected with Cas9-endonuclease and sgRNAs targeting the top DUB candidates obtained from the initial

PR-619 was used as a positive control. Among the DUBKO library-based putative DUB candidates, USP3 was the strongest candidate for regulating $\mathrm{Cdc} 25 \mathrm{~A}$ protein stability (Fig. 2d). Next, we checked the level of Cdc25A protein upon transient knockout and knockdown of USP3 in HeLa cells via the CRISPR-Cas9 system and shRNA lentiviral particles targeting USP3, respectively. The data demonstrate that depletion of USP3 by either shRNA or sgRNA causes a significant downregulation in $\mathrm{Cdc} 25 \mathrm{~A}$ protein turnover (Fig. 2e). The overexpression of USP3 (Fig. 3a, lane 3) but not USP3CS (Fig. 3a, lane 4) increased the level of the Cdc25A protein. In addition, depletion of USP3 by either shRNA or sgRNA downregulated Cdc25A protein
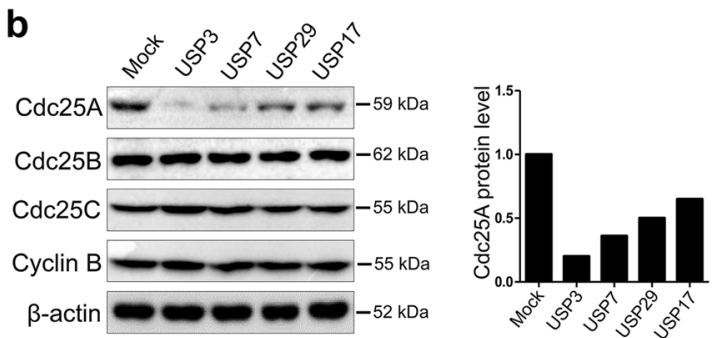

C
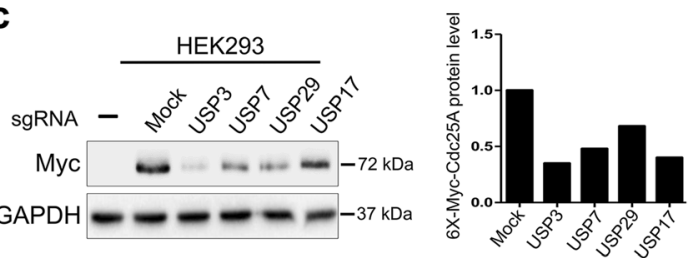

d
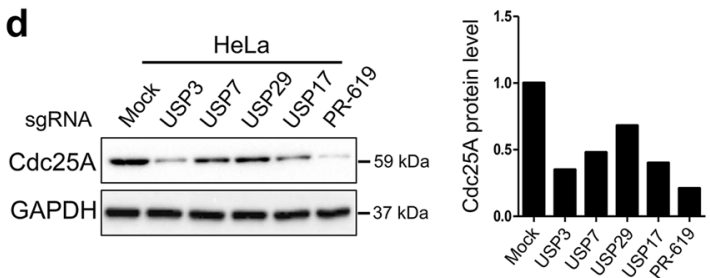

e

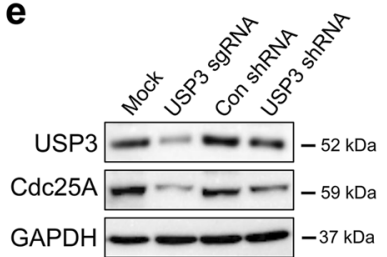

screening. Exogenous Cdc25A expression was assessed using an antiMyc antibody. The graph in the right panel represent the expression of the $6 \times-\mathrm{Myc}-\mathrm{Cdc} 25 \mathrm{~A}$ protein as estimated by ImageJ software. $\mathbf{d} \mathrm{HeLa}$ cells were transiently transfected with the sgRNAs for the putative top DUB candidates and Cas9, and endogenous Cdc25A was analyzed. As a positive control, HeLa cells were incubated with $10 \mathrm{nM}$ PR-619, a common DUB inhibitor. The graph in the right panel represent the expression of the Cdc25A protein as estimated by ImageJ software. e Western blot of endogenous USP3 and Cdc25A in HeLa cells transfected with non-targeted sgRNA (mock) or sgRNA targeting USP3 or transduced with control shRNA or USP3 shRNA lentiviral particles.

expression (Fig. 3a, lanes 5 and 6). We further demonstrated that the expression of USP 3 but not USP3CS could stabilize Cdc25A protein levels in a dose-dependent manner (Fig. 3b and Supplementary Fig. S7a).

We further quantitatively analyzed the effect of USP3 on Cdc25A stabilization using a dual fluorescence [Discosoma red fluorescent protein (DsRed) and enhanced green fluorescent protein (EGFP)] tagged reporter plasmid system encoding the Cdc25A gene. Using that system, any event that affects the stability of the Cdc25A protein could be monitored by the changes in the DsRed/EGFP ratio in a flow-cytometry analysis [21]. The DsRed signal indicates the transfection efficiency, and the expression of EGFP is 


\section{a}

6X-Myc-Cdc25A -+++++

Flag-USP3 --+--

Flag-USP3CS ---+-

USP3-shRNA ----+

USP3-sgRNA -----+

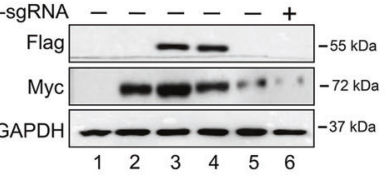

d

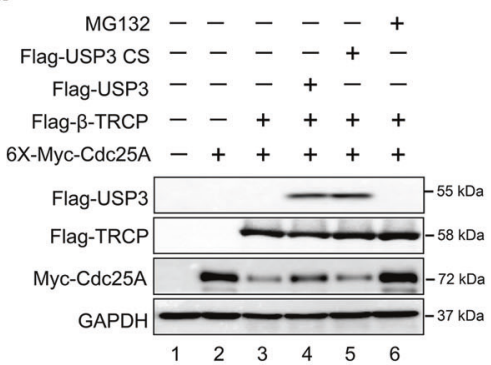

g

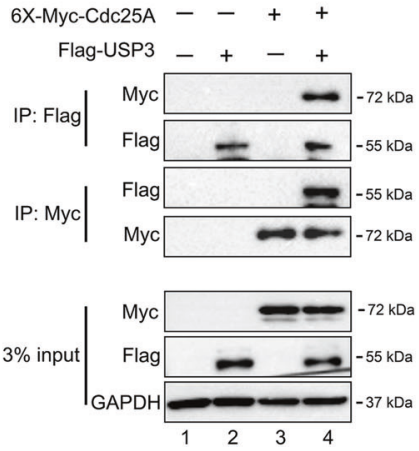

b

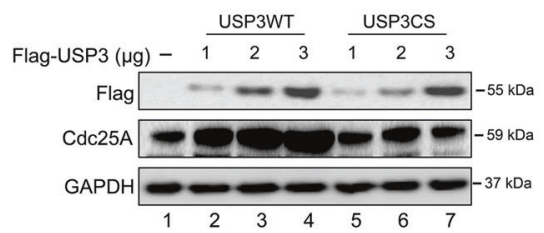

e

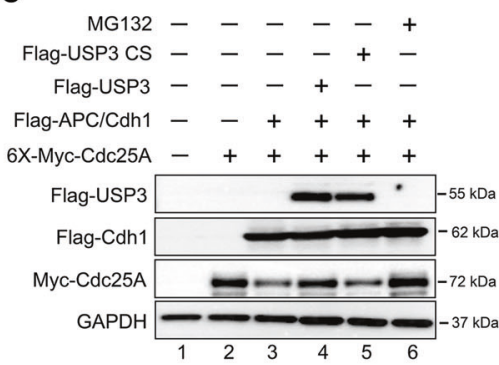

h

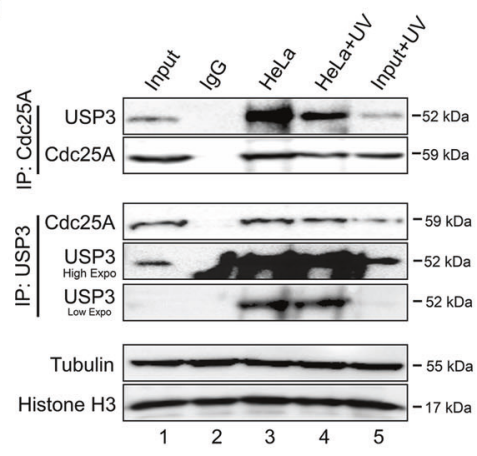

C

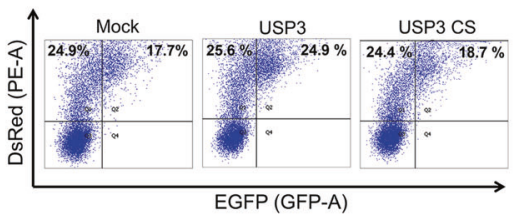

f

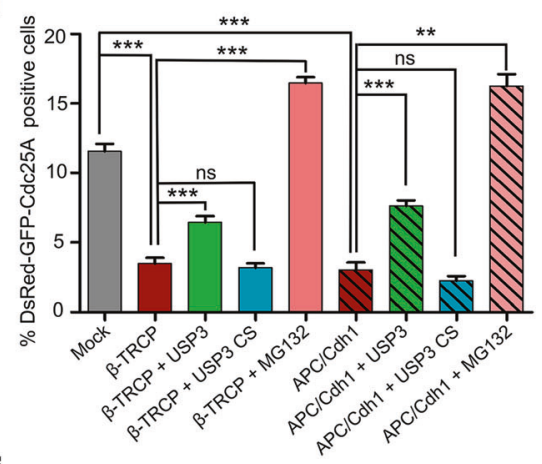

i

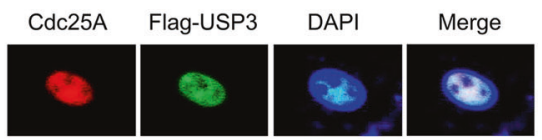

Myc-Cdc25A USP3
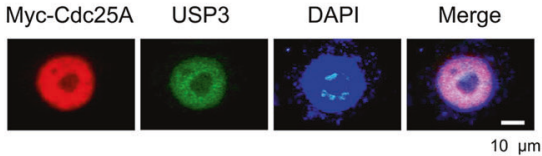

Fig. 3 USP3 interacts with Cdc25A and regulates Cdc25A stability. a HEK293 cells were transfected with $6 \times-$ Myc-Cdc25A alone or in combination with Flag-USP3, Flag-USP3CS, and shRNA or sgRNA targeting USP3. Western blot analyses were performed to determine the exogenous Cdc25A protein level. b The endogenous Cdc25A protein level in HeLa cells was analyzed upon transfection with increasing concentrations of Flag-USP3 or Flag-USP3CS. $\mathbf{c}$ The change in the pAd-DsRed-IRES-EGFP-Cdc25A signal in the presence of either Flag-USP3 or Flag-USP3CS was estimated by flowcytometry analysis. d-e The effect of E3 ligases, $\beta$-TRCP, and APC/ Cdh1 on exogenous Cdc25A stability was determined in the presence and absence of Flag-USP3 and Flag-USP3CS by transiently transfecting HEK293 cells with the respective constructs. To block the effect of the E3 ligases on Cdc25A, MG132 was applied for $6 \mathrm{~h}$. $\mathbf{f}$ The effect of E3 ligases ( $\beta$-TRCP and APC/Cdh1) on DsRed/EGFP-tagged $\mathrm{Cdc} 25 \mathrm{~A}$ in the presence and absence of Flag-USP3, Flag-USP3CS, and MG132 was determined by transiently transfecting HEK293 cells

directly proportional to the Cdc25A protein level. We cotransfected HEK293 cells with USP3 or USP3CS and the Cdc25A reporter plasmid and monitored the EGFP expression by flow cytometry and western blot analyses. The DsRed/EGFP signal ratio showed a significant increase in response to USP3 but not the catalytic mutant (Fig. 3c and Supplementary Fig. S7b), while the DsRed with the respective constructs and a reporter plasmid encoding pAdDsRed-IRES-EGFP-Cdc25A. The DSRed/EGFP signal was estimated by flow-cytometry analysis. The data shown here are the mean $\pm \mathrm{SD}$ of three independent experiments $(* * P<0.005$, $* * * P<0.0005$, and ns denotes non-significant). $g$ The exogenous interaction between Flag-USP3 and Myc-Cdc25A in HEK293 cells was evaluated by coimmunoprecipitation assays. h Co-immunoprecipitation of endogenous USP3 and Cdc25A from HeLa cells. The indicated cells were treated with UV $\left(20 \mathrm{~J} / \mathrm{m}^{2}\right)$. $\beta$-Tubulin and histone $\mathrm{H} 3$ were used as cytoplasmic and nuclear markers, respectively. i An immunofluorescence staining assay was performed to analyze the localization patterns of USP3 and Cdc25A in HeLa cells. Because specific antibodies against USP 3 and Cdc25A are derived from the same species, we transfected HeLa cells with either Flag-USP3 or Myc-Cdc25A and then performed staining with Flag- and Cdc25A-specific antibodies or Myc- and USP3-specific antibodies. Scale bar, $10 \mu \mathrm{m}$.

signal remained constant (Fig. 3c). A similar increase in EGFP-Cdc25A expression upon co-transfection with FlagUSP3 was observed in the western blots (Supplementary Fig. S7c).

$\mathrm{Cdc} 25 \mathrm{~A}$ is unstable during the $\mathrm{S}$ phase and is degraded through the $\beta$-TRCP and APC/Cdh1-mediated ubiquitinproteasome pathways $[7,22]$. The destabilizing effect of 
$\beta$-TRCP and APC/Cdh1 on Cdc25A was counteracted by USP3 or the proteasomal inhibitor MG132 but not USP3CS (Fig. 3d, e, lanes 4-6), which we further quantified by comparing the change in the DsRed/EGFP signal ratio via flow cytometry (Fig. 3f and Supplementary Fig. S7d) and changes in the GFP-Cdc25A expression level via western blot analyses (Supplementary Fig. S7e).

\section{USP3 interacts with Cdc25A and co-localizes with Cdc25A in the nucleus}

Next, we demonstrated that Flag-USP3 and 6x-MycCdc25A interact with each other in HEK293 cells (Fig. 3g). Similarly, ectopically expressed Flag-USP3 in HeLa cells was found to co-precipitate endogenous Cdc25A and vice versa (Supplementary Fig. S8a). A previous report showed that $\mathrm{Cdc} 25 \mathrm{~A}$ undergoes rapid degradation in response to genotoxic stress such as ultraviolet (UV) exposure [23]. Thus, we next investigated the interaction between endogenous USP3 and Cdc25A in the presence and absence of UV-based DNA damage. Our results indicate that endogenous USP3 and Cdc25A interact with each other in both conditions (Fig. 3h). Further, USP3 showed nuclear co-localization with Cdc25A in HeLa cells (Fig. 3i). In addition, USP3 was found to be co-localized with Cdc25A at both the $\mathrm{S}$ phase and $\mathrm{M}$ phase of the cell cycle (Supplementary Fig. S8b).

\section{USP3 deubiquitinates and extends Cdc25A protein half-life}

In line with previous reports [24-26], endogenous Cdc25A was found to be directly proportional to the dose of MG132, indicating that Cdc25A undergoes 26S proteasomal degradation (Supplementary Fig. S8c). To further support our result, we used the TUBEs assay, which is a high affinity probe for ubiquitinated proteins [27, 28]. Our data confirm that $\mathrm{Cdc} 25 \mathrm{~A}$ undergoes rapid ubiquitination when $\mathrm{HeLa}$ cells are stimulated with IL- $1 \beta$, compared with nonstimulated cells (Supplementary Fig. S8d). Next, we checked the effect of USP3 on the Cdc25A mRNA level. The RT-PCR data reveal that USP3 does not regulate the transcription of Cdc25A (Supplementary Fig. S8e). Therefore, we speculate that USP3 might function as a deubiquitinase regulating $\mathrm{Cdc} 25 \mathrm{~A}$ protein stability. To this end, we examined the polyubiquitination of $\mathrm{Cdc} 25 \mathrm{~A}$ in the presence of USP3 or the catalytic mutant USP3CS, along with the exogenous expression of ubiquitin in HEK293 cells (Fig. 4a). We also analyzed endogenous Cdc25A polyubiquitination in the presence of USP3, USP3CS, or USP3 shRNA in HeLa cells (Fig. 4b). Overexpression of USP3 led to a decrease in both exogenous and endogenous $\mathrm{Cdc} 25 \mathrm{~A}$ polyubiquitination (Fig. 4a lane 4 and Fig. 4b lane 3), but this reduction was not found in response to USP3CS (Fig. 4a lane 5 and Fig. 4b lane 4) or upon knockdown of USP3 (Fig. 4b, lane 5). A similar reduction in the level of polyubiquitinated Cdc25A was observed in the presence of USP3 but not USP3CS (Fig. 4c, lanes 3 and 4).

Further, the deubiquitinating activity of USP3 extended the half-life of endogenous Cdc25A (Fig. 4d, lanes 4-6). Interestingly, USP3 knockout in HEK293 cells caused a severe reduction in the half-life of endogenous $\mathrm{Cdc} 25 \mathrm{~A}$ compared with the mock control cells (Fig. 4e, lanes 4-6), whereas treating the mock control cells with the proteasome inhibitor MG132 led to accumulation of Cdc25A protein (Fig. 4e, lanes 7-9). We further confirmed our results by analyzing the half-life of exogenous Cdc25A in the presence of Flag-USP3 or upon treatment with the proteasomal inhibitor MG132. Overexpression of USP3 extended the half-life of exogenous Cdc25A (Supplementary Fig. S8f).

\section{USP3 overexpression stimulates DNA damage response}

We next investigated the role of USP3 in cell-cycle progression by overexpressing USP3 or USP3CS along with shRNA-mediated USP3 knockdown in HeLa cells. The transfected samples were confirmed by western blot (Supplementary Fig. S8g), and the same batch of transfected cells was subjected to cell-cycle distribution analysis by PI staining. The results show a significant accumulation of cells in the S phase when USP3 is overexpressed, but not USP3CS (Fig. 5a). In contrast, USP3-silenced cells showed G0/G1 phase accumulation, indicating an impairment of the G1-S phase transition compared with the mock control (Fig. 5a). Cdc25A undergoes rapid, ubiquitin-mediated proteasomal degradation in response to DNA damage by genotoxic stress such as UV light, leading to checkpoint activation and consecutive inhibition of DNA replication [23]. The activation of oncogenes such as Cdc25A causes DNA replication stress that induces DNA damage and cancer progression. Thus, we predicted that overexpression of USP3 might replicate that effect by stabilizing Cdc25A. Interestingly, we observed a significant increase in phosphorylated histone-H2AX immune-positive cells, which are a marker for double-stranded DNA breaks, in USP3overexpressing cells compared with the mock control (Fig. 5b). In addition, upon genotoxic stress with etoposide, USP3-overexpressing cells showed less apoptosis, whereas USP3-silenced cells showed more apoptosis (as measured by DNA content at the sub-G1 phase) than the mock controls (Fig. 5c). 
a

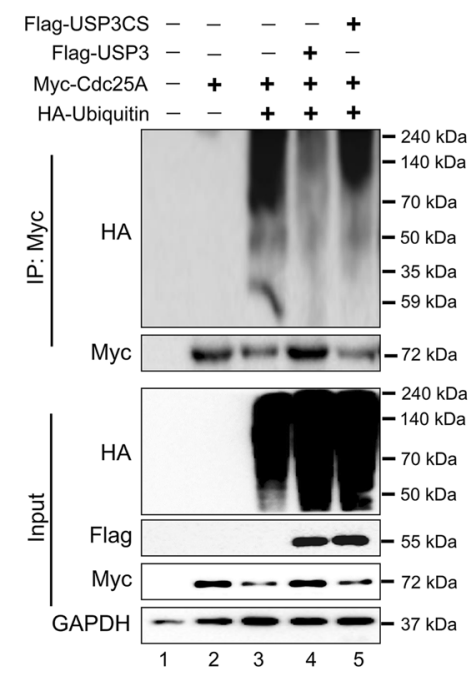

b
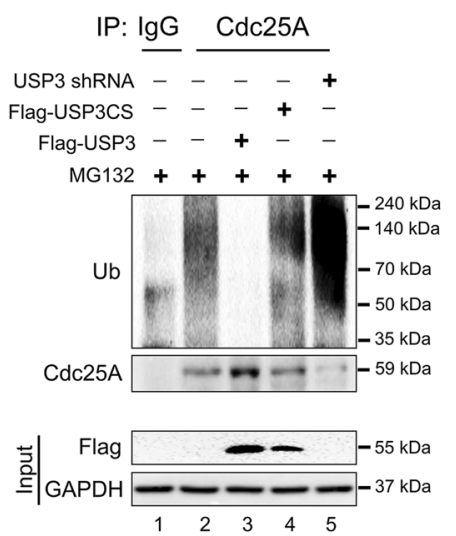

C

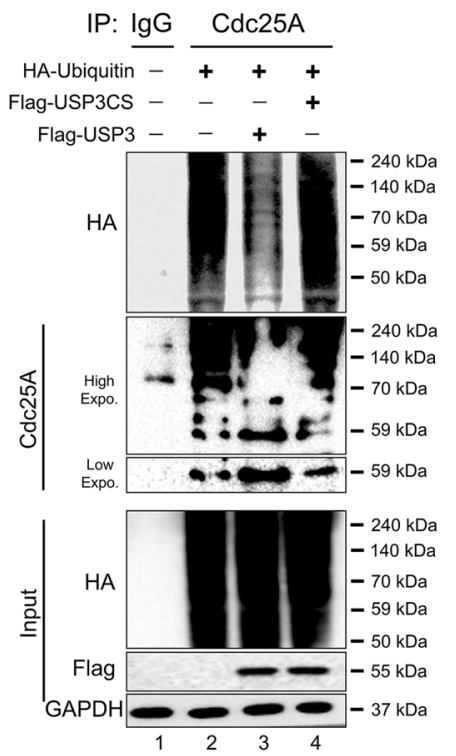

d
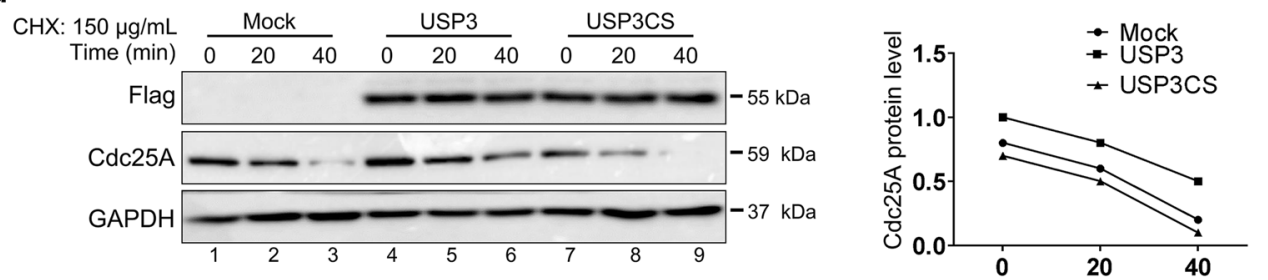

e
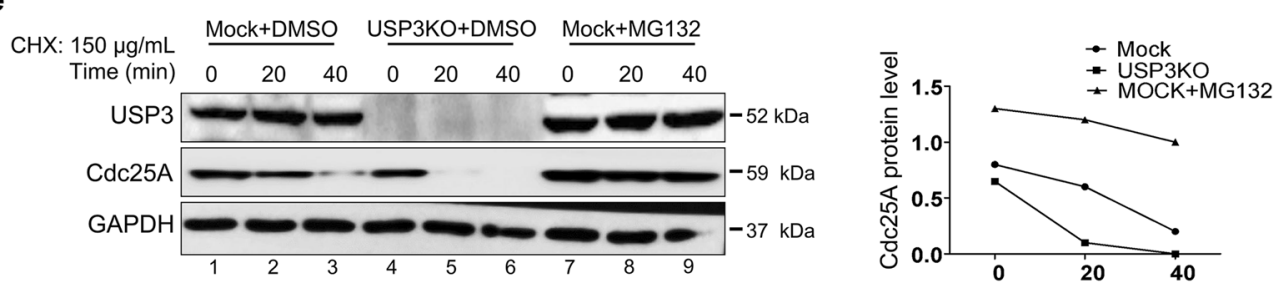

Fig. 4 USP3 deubiquitinates Cdc25A and extends the half-life of Cdc25A protein. a Myc-Cdc25A and HA-ubiquitin were cotransfected into HEK293 cells with Flag-USP3 or Flag-USP3CS. The cells were immunoprecipitated with Myc antibody, followed by immunoblotting with HA antibody. b HeLa cells transfected with Flag-USP3 or Flag-USP3CS or transduced with USP3 shRNA were treated with MG132 $(5 \mu \mathrm{M})$ for $4 \mathrm{~h}$, and the polyubiquitination of endogenous Cdc25A was examined. Immunoprecipitation was performed with Cdc25A-specific antibody, followed by immunoblotting with ubiquitin antibody. c HeLa cells were transfected with FlagUSP3, Flag-USP3CS, and HA-ubiquitin, and the polyubiquitination of endogenous $\mathrm{Cdc} 25 \mathrm{~A}$ was examined. Immunoprecipitation was

\section{Generation of single cell-derived knockout clones of USP3 and Cdc25A in HeLa cells}

Given that Cdc25A removes inhibitory phosphorylation in CDKs and plays a major role in the G1-to-S phase transition $[29,30]$, we examined the role of USP3 in the cell-cycle progression of $\mathrm{HeLa}$ cells by generating a single cellderived USP3KO and $\mathrm{Cdc} 25 \mathrm{KO}$ clones. We designed two sgRNAs specific to exon 1 and exon 3 in USP3 performed with Cdc25A-specific antibody, followed by immunoblotting with anti-HA antibody. d HEK293 cells transfected with empty vector (mock), Flag-USP3, or Flag-USP3CS were treated with cycloheximide (CHX) $(150 \mu \mathrm{g} / \mathrm{mL})$ for the indicated times. Cells were harvested for immunoblotting with the indicated antibodies. The expression of endogenous Cdc25A protein was estimated by ImageJ software and is represented graphically in the right panel. e Mock control and USP3KO HEK293 cells were incubated with CHX for the indicated times. Cells were treated with either DMSO (control) or MG132 $(5 \mu \mathrm{M})$ for $4 \mathrm{~h}$ prior to CHX treatment. The expression of endogenous Cdc25A protein was estimated by ImageJ software and is represented graphically in the right panel.

(Supplementary Fig. S9a, left panel) and two sgRNAs targeting exon 1 of Cdc25A (Supplementary Fig. S10a, left panel). We assessed the knockout efficiency of sgRNA1 and sgRNA2 in a pooled batch of transfected HeLa cells using USP3 and Cdc25A-specific antibodies (Supplementary Figs. S9a and S10a, right panel). The pooled transfected cells were diluted and seeded into 96-well plates. Single cell-derived clones were then screened for USP3 and $\mathrm{Cdc} 25 \mathrm{~A}$ genes disruption by the T7E1 assay 
a
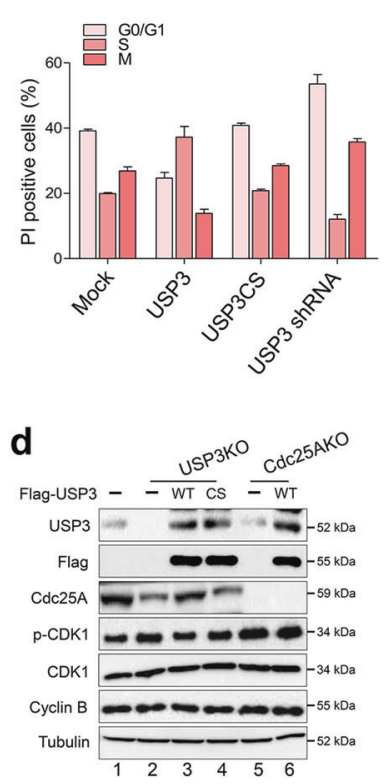

f

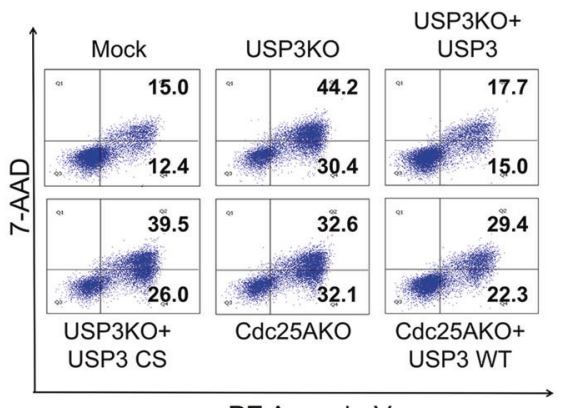

PE Annexin-V

e b

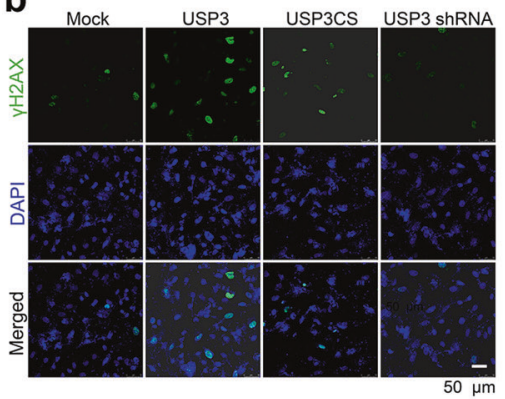

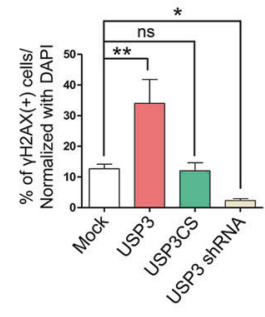

C
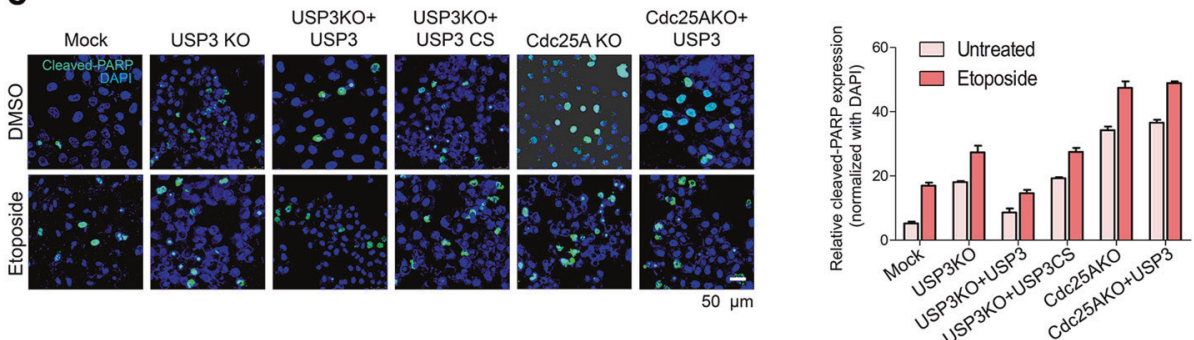

g
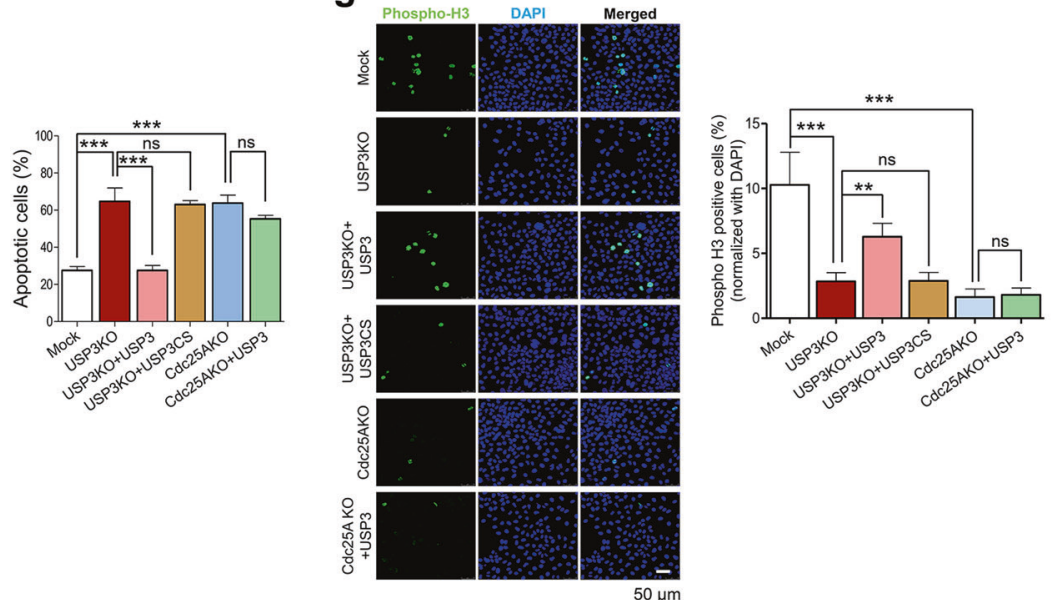

h
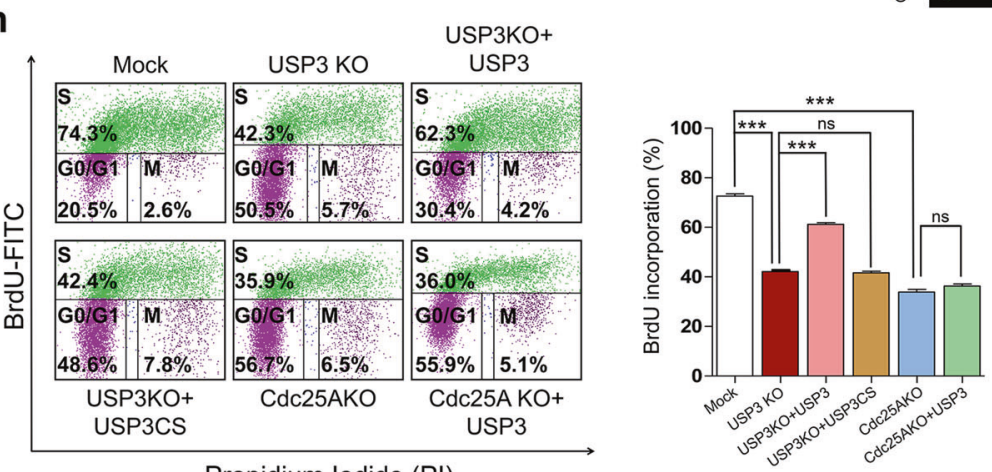

i
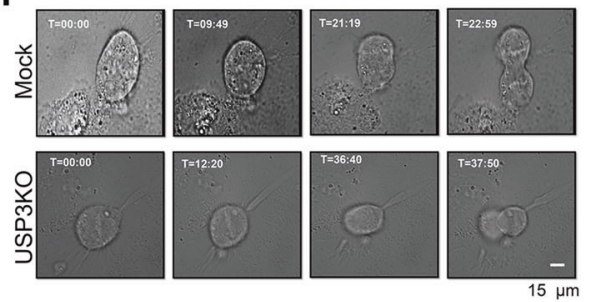

Propidium lodide $(\mathrm{PI})$

(Supplementary Figs. S9b and S10b). The T7E1-positive single cell-derived USP3 and Cdc25A knockout clones showing a high indel percentage were subjected to Sanger sequencing to confirm gene disruption (Supplementary Figs. S9c and S10d), and the protein expressions of USP3 and Cdc25A were abolished in USP3KO\#8 and
Cdc25AKO\#10 cell lines (Supplementary Figs. S9d and S10c). Particularly, USP3KO\#8 showed a significant decrease in Cdc25A level compared with controls (Supplementary Fig. S9d). An off-target analysis of USP3KO\#8 and Cdc25AKO\#10 showed no non-specific cleavages (Supplementary Figs. S9e and S10e). We therefore used 
Fig. 5 Loss of USP3 destabilizes Cdc25A, leading to cell-cycle arrest. a The cell-cycle status of HeLa cells transfected with FlagUSP3 or Flag-USP3CS and USP3-silenced cells based on their DNA content in a flow-cytometry analysis. The data presented here are the mean \pm SD of three independent experiments. b The indicated cells were further stained with a DNA damage marker, phosphorylated $\mathrm{H} 2 \mathrm{AX}(\gamma \mathrm{H} 2 \mathrm{AX}$, green staining). Blue staining indicates DAPI-labeled nuclei. At least 200 cells per group from three independent experiments were examined and are graphically represented $(* P<0.05$, $* * P<0.005$, and ns denotes non-significant). Scale bar, $50 \mu \mathrm{m}$. c FlagUSP3- or Flag-USP3CS-transfected HeLa cells and USP3 knockdown cells were treated with etoposide for $12 \mathrm{~h}$ and subjected to PI staining to quantify sub-diploid G1 content. The data presented here are the mean \pm SD of three independent experiments. d USP3KO HeLa cells were reconstituted with ectopic expression of WT-USP3 or USP3CS, and Cdc25AKO HeLa cells were transfected with WT-USP3, followed by immunoblotting with p-CDK1 and the other indicated antibodies. e The above-mentioned cells were immunostained with cleaved-PARP antibody to determine the population of apoptotic cells upon treatment with DMSO or etoposide. The cells expressing cleaved-PARP (green fluorescence) were counted and normalized to the DAPI/nuclei (blue fluorescence). At least 400 cells per group from three independent experiments were examined and are graphically represented in the right panel. Scale bar, $50 \mu \mathrm{m}$. f Cells were used to estimate the percentage of apoptotic cells by PE annexin-V/7-AAD staining. The data presented here are the mean $\pm \mathrm{SD}$ of three independent experiments (*** $P<0.0005$ and $\mathrm{ns}$ denotes non-significant). $\mathbf{g}$ The indicated cells were further immunostained with phospho-H3 (Ser10) antibody. The cells expressing phospho-H3 (green fluorescence) were counted and normalized to DAPI/nuclei staining (blue fluorescence). Scale bar, $50 \mu \mathrm{m}$. At least 400 cells per group from three independent experiments were examined and are graphically represented. The data presented here are the mean $\pm \mathrm{SD}$ of three independent experiments $(* * P<0.005, * * * P<0.0005$, and ns denotes non-significant). $\mathbf{h}$ The indicated asynchronously growing cells were pulsed with BrdU for $1 \mathrm{~h}$ and then subjected to a flow-cytometry analysis to measure BrdU incorporation into DNA. The data presented here are the mean \pm SD of three independent experiments $(* * * P<0.0005$ and ns denotes nonsignificant). i GFP-H2B-expressing USP3KO or mock control U2OS cells were observed using time-lapse microscopy for $12 \mathrm{~h}$. Images were captured every $1 \mathrm{~min}$. Scale bar, $15 \mu \mathrm{m}$.

USP3KO\#8 (hereafter called USP3KO) and Cdc25AKO\#10 (hereafter called Cdc25AKO) for further functional studies. HeLa cells co-transfected with Cas9 and scrambled sgRNA were used as the vector control (mock).

\section{USP3 regulates the Cdc25A-mediated cell-cycle transition in HeLa cells}

Cdc25A phosphatase activates Cdk1 by removing inhibitory phosphatases from the $\mathrm{Thr}^{14}$ and $\mathrm{Thr}^{15}$ residues [31]. To investigate the role of USP3 in Cdc25A's regulation of Cdk1, we reconstituted USP3KO cells with either USP3 or USP3CS, and Cdc25AKO cells were overexpressed with USP3. The results show a rescue of endogenous Cdc25A upon USP3 overexpression (Fig. 5d, lane 3) compared with USP3KO cells (Fig. 5d, lane 2) but not upon expression of USP3CS (Fig. 5d, lane 4). Furthermore, we found an upregulation in the level of phospho-Cdk1 (p-CdK1) upon the loss of USP3 (Fig. 5d, lane 2), and reconstituting
USP3KO with USP3 (but not USP3CS) caused a reduction in the level of p-CdK1 (Fig. 5d, lanes 3 and 4). On the other hand, Cdc25AKO cells showed more phosphorylation of Cdk1 than USP3KO cells (Fig. 5d, lane 5), and overexpressing USP3 in Cdc25AKO cells did not alter the expression of p-Cdk1 compared with Cdc25AKO cells (Fig. 5d, lane 6), indicating that the expression of Cdc25A is critical to the level of Cdk1 phosphorylation.

The same batch of cells were treated with etoposide and subjected to a colony survival assay. The colony numbers decreased severely upon USP3 knockout, and reconstitution of USP3 (but not USP3CS) caused an increase in colony numbers (Supplementary Fig. S11a, b). In contrast, Cdc25AKO cells showed a severe decrease in colony numbers compared with USP3KO cells, and overexpressing USP3 in Cdc25AKO cells did not produce any significant changes in colony numbers (Supplementary Fig. S11a, b), suggesting that Cdc25A is a critical factor for cell growth, proliferation, and survival. We treated the same batches of cells with either DMSO or etoposide and then immunostained them with an apoptotic marker (cleaved-PARP). We found more apoptotic USP3KO cells and Cdc25KO cells than mock control cells (Fig. 5e). In addition, when we stained these cells with $\mathrm{PE}$ annexin-V/7-AAD, we also found more apoptotic USP3KO cells than mock control cells (Fig. 5f).

Next, we immunostained the cells with a phospho-H3 (Ser10) antibody that binds with mitotic chromosomes when histone $\mathrm{H} 3$ is phosphorylated. A reduction in the expression of phospho-H3 was observed in the USP3KO cells compared with the mock control, and the decrease was even more severe in the Cdc25AKO cells (Fig. 5g). Consistent with these results, the USP3KO cells incorporated less BrdU than the mock controls, indicating that fewer cells had entered the $\mathrm{S}$ phase, and reconstitution of USP3 (but not USP3CS) led to an upregulation in BrdU incorporation (Fig. 5h). We next synchronized the mock cells and USP3KO cells at mitosis with nocodazole for mitotic arrest. The USP3KO cells showed a relatively slow mitotic exit upon mitotic release (Supplementary Fig. S12). In addition, we monitored the cell division of mock and USP3-deficient cells at defined times using time-lapse microscopy. The cell division time was delayed by USP3 knockdown (Fig. 5i, Supplementary movie S2) compared with the mock control (Fig. 5i, Supplementary movie S1), further indicating that USP3 plays a role in maintaining the $\mathrm{Cdc} 25 \mathrm{~A}$ stability required for proper cell-cycle progression.

\section{USP3 regulates the oncogenic potential of Cdc25A}

In mammalian cells, replication stress is sensitized and suppressed by a signaling cascade led by the ATR kinase [32,33]. 
Recently, a genome-wide screening report showed that $\mathrm{Cdc} 25 \mathrm{~A}$ is abundantly expressed in tumors that are sensitive to ATR inhibitors, and Cdc25A deficiency renders mammalian cells resistant to ATR inhibitors [34]. Therefore, we speculated that USP3KO cells would show similar resistance to ATR inhibitors due to Cdc25A destabilization. To test that idea, we treated mock control, USP3KO, USP3KO reconstituted with USP3 or USP3CS, Cdc25AKO, and Cdc25AKO cells overexpressed with USP3 with ATR inhibitors (caffeine and AZ20). Notably, the USP3KO and Cdc25AKO cells showed significant resistance to high doses of ATR inhibitors, whereas reconstitution of USP3KO cells with USP3 (but not USP3CS) induced sensitivity to ATR inhibitors (Fig. 6a). To corroborate the role of USP3-promoted Cdc25A stabilization in cellular proliferation, the cells were subjected to various assays examining carcinogenesis-related activity. USP3KO and Cdc25AKO cells showed a significant reduction in cell viability (Fig. 6b), invasion rate (Fig. 6c and Supplementary Fig. S13a), and migration (Fig. 6d and Supplementary Fig. S13b) compared with the mock control. However, rescue of USP3 in USP3KO cells caused an increase in carcinogenic behavior (Fig. 6b-d).

Further, we performed anchorage-independent colony formation assays. Those results show that knockout of USP3 and Cdc25A impeded the colony formation of HeLa cells (Fig. 6e). In line with our previous observations, these results indicate that USP3 has a Cdc25A-dependent carcinogenic function in human cervical cancer cells (Fig. 6e). To further corroborate the effect of USP3 on Cdc25A-dependent oncogenic transformation, we generated USP3KO cells stably expressing $\mathrm{Cdc} 25 \mathrm{~A}$ and analyzed by western blot (Fig. 6f). We subcutaneously transplanted those cells into NSG mice and recorded the tumor growth every other day for 4 weeks. In the NSG mice transplanted with USP3KO cells, the tumor growth was significantly suppressed compared with the mice that received mock control cells, whereas the overexpression of $\mathrm{Cdc} 25 \mathrm{~A}$ in USP3KO cells led to a significant increase in tumor volume (Fig. 6g), and weight (Fig. 6h). Furthermore, our immunohistological analysis of the tumor tissues showed that both USP3 and Cdc25A levels were significantly decreased in USP3KO tumor samples compared with the mock controls (Fig. 6j).

\section{Evaluation of USP3 and Cdc25A as cancer biomarkers}

We next examined the expression levels of Cdc25A and USP3 individually using TCGA data in several cancer types for which normal-tumor matched RNAseq expression data were available. Cdc25A was significantly upregulated in $75 \%$ (15 of 20) of cancer types (Supplementary Fig. S14), and USP3 was significantly upregulated in 35\% (7 of 20) of cancer types (Supplementary Fig. S15). The TCGA cohorts showed a possible regulatory link between the expression levels of Cdc25A and USP3 because $85.7 \%$ of the cancer types (6 of 7) that showed higher expression of USP3 in tumor tissues also showed higher expression of $\mathrm{Cdc} 25 \mathrm{~A}$ in tumor tissues (Fig. 7a and Supplementary Figs. S14 and S15). Among them are some of the most prevalent cancer types, including breast invasive cancer (BRCA), liver hepatocellular carcinoma (LIHC), lung adenocarcinoma (LUAD), prostate adenocarcinoma (PRAD), bladder urothelial carcinoma (BLCA), cholangiocarcinoma, and testicular germ cell tumors demonstrating a significant correlation between Cdc25A-USP3 upregulation and tumorigenesis. Likewise, immunoblotting showed that the expression of Cdc25A correlated positively with USP3 expression in several human cancer cell lines (Fig. 7b).

We next used a Kaplan-Meier analysis of TCGA survival data to investigate whether elevated expression levels of USP3 and Cdc25A could serve as a prognostic marker predicting patient survival. Out of 32 tumor types with OS data available, the patient group with the highest expression of Cdc25A was significantly associated with worse survival in 9 tumor types [acute myeloid leukemia (ACC), lowergrade glioma (LGG), LIHC, LUAD, kidney chromophobe, sarcoma, kidney renal clear cell carcinoma, mesothelioma, and skin cutaneous melanoma and with better survival in only 3 tumor types (uveal melanoma, colon adenocarcinoma, and stomach adenocarcinoma)] (Supplementary Fig. S16). On the other hand, high expression of USP3 was associated with worse survival in three tumor types [ACC, BRCA, and pancreatic adenocarcinoma] and better survival in two brain tumor types [LGG and glioblastoma multiforme (GBM)] (Supplementary Fig. S17). Notably, high expression of both Cdc25A and USP3 was associated with poor survival in ACC (Supplementary Figs. S16 and S17).

Finally, we explored the possibility that USP3 and Cdc25A could act as biomarkers in breast cancer because (1) Cdc25A has been identified as a therapeutic target in early stage breast cancer and triple negative breast cancers (36, 37), (2) high expression of Cdc25A was associated with poor patient survival in a compendium of breast cancer patients [35, 36], and (3) high expression of USP3 correlated with a poor survival rate in breast cancer patients according to our analysis $(P=0.0264)$ (Fig. 7c). We confirmed experimentally (western blotting) that the expression of Cdc25A and USP3 was strongly correlated in several breast cancer cell lines (Fig. 7d). Furthermore, we investigated USP3 and Cdc25A expression in 12 breast cancer tissues obtained from the ISU ABXIS cohort and found that both proteins were frequently upregulated in these tumors (Fig. 7e and Supplementary Fig. S18). Together with the fact that both Cdc25A and USP3 are upregulated in BRCA, these results suggest that the Cdc25A-USP3 axis could 
a

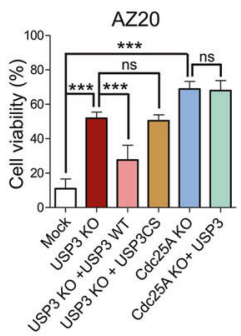

e

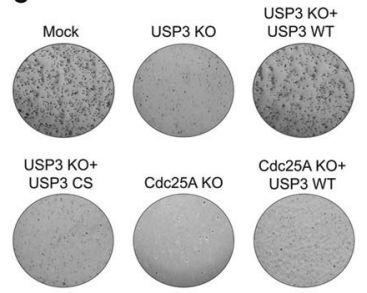

h

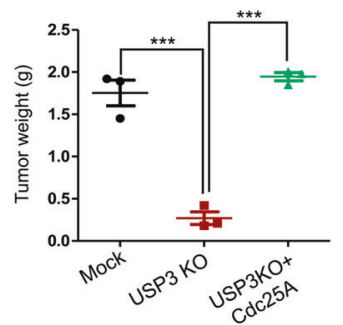

b

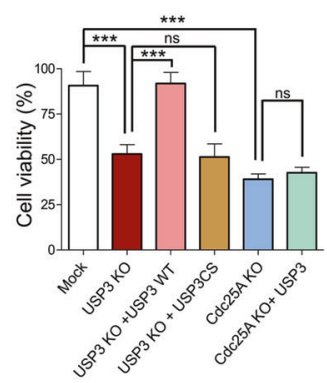

C

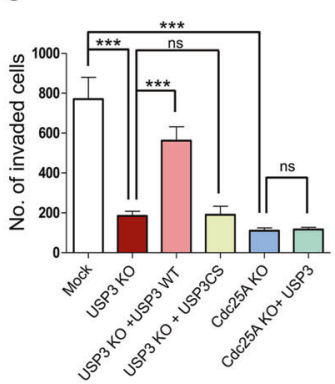

d

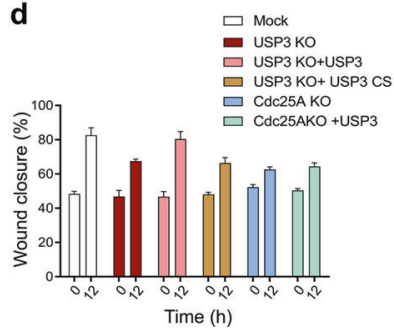

f

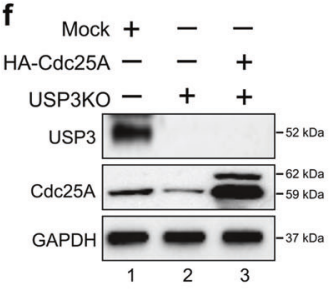

j

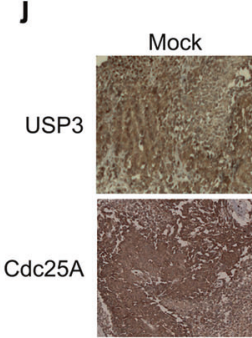

g

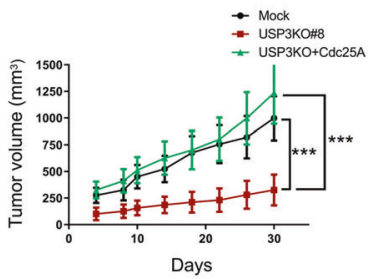

i

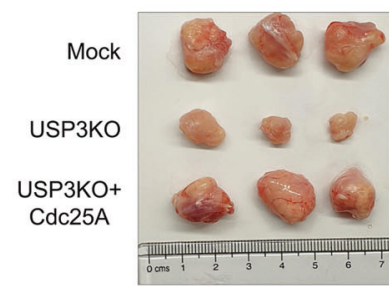

Fig. 6 The role of USP3 in the pathogenesis of cancer. a To evaluate the role of USP3 in Cdc25A-mediated sensitivity to ATR inhibitors, empty vector (mock) HeLa cells, USP3KO cells, USP3KO cells reconstituted with USP3 or USP3CS, Cdc25AKO cells, and Cdc25AKO cells overexpressed with USP3 were treated with AZ20 or caffeine for $48 \mathrm{~h}$ and then subjected to the CCK- 8 cell proliferation assay. The data presented here are the mean \pm SD of three independent experiments $(* * * P<0.0005$ and $\mathrm{ns}$ denotes non-significant). b The cell proliferation rate in the above-mentioned cells was assessed by CCK- 8 assay. The data presented here are the mean \pm SD of three independent experiments $(* * * P<0.0005$ and ns denotes non-significant). c In vitro invasion ability was examined using a Transwell invasion assay. The data presented here are the mean $\pm \mathrm{SD}$ of three independent experiments $(* * * P<0.0005$ and ns denotes non-significant). d A wound healing assay was performed to evaluate the migration of the indicated cells. Cells were cultured in fetal bovine serum-free medium during the experimental period, and the wound closure percentage was calculated. The area of the wound was estimated by Image J software at 0 and $12 \mathrm{~h}$ and is represented graphically.

serve as a prognostic marker and novel regulatory target in breast cancer.

\section{Discussion}

Our genome-scale loss-of-function screening library for functional DUBs from individually generated USP knockout mammalian cell lines provides a method that is
The data presented here are the mean \pm SD of three independent experiments. e A soft-agar colony formation assay was performed using the indicated cells. The numbers of colonies were quantified and are represented graphically. The data presented here are the mean $\pm \mathrm{SD}$ of three independent experiments $(* * * P<0.0005$ and ns denotes nonsignificant). f Mock, USP3KO, and HA-Cdc25A stably expressing USP3KO HeLa cells were analyzed by immunoblotting with the indicated antibodies. $g$ The volume of subcutaneous tumor xenografts from mock, USP3KO, and HA-Cdc25A-expressing USP3KO HeLa cells was measured every other day after injection. Data points represent the mean $\pm \mathrm{SD}$ of five mice per group $(* * * P<0.0005$ and ns denotes non-significant). $\mathbf{h}$ The tumor weight from each mouse was recorded and is represented graphically. The data presented here are the mean $\pm \mathrm{SD}$ of three independent experiments $(* * * P<0.0005$ and ns denotes non-significant). i Representative xenograft tumors are shown. $\mathbf{j}$ Tumor tissue derived from mouse xenografts was subjected to immunohistochemical staining with USP3- and Cdc25A-specific antibodies. Scale bar $=150 \mu \mathrm{m}$.

mechanically distinct from RNAi and shRNA-based DUB screening. The main difference between the approaches is that the RNAi and shRNA-based methods often cause partial repression or restoration of gene function in a few generations and are temporary, whereas DUBKO introduces a complete loss-of-function mutation into genomic DNA, and its effect is permanent. In this study, we used the CRISPR-Cas9 technique to individually target genes for all members of a DUB subfamily. We then generated single 
a

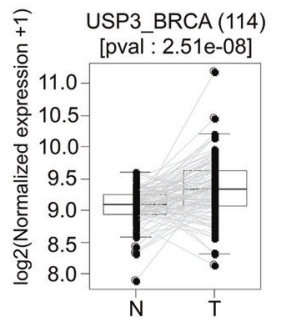

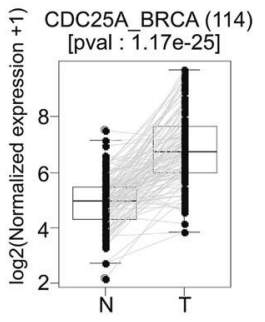

b

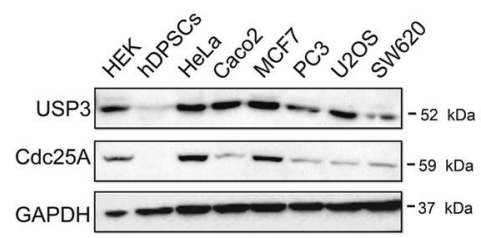

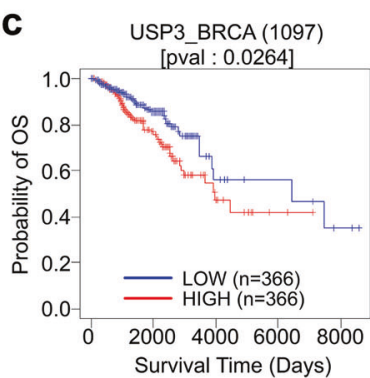

d

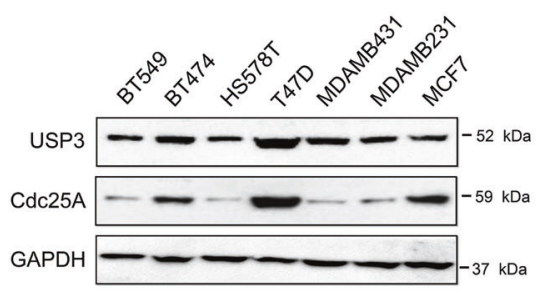

e

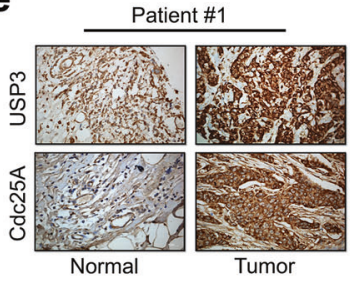

Patient \#4
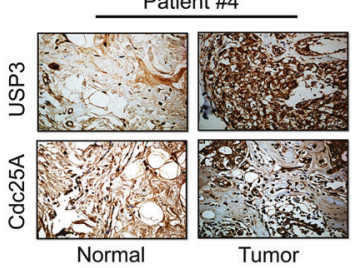

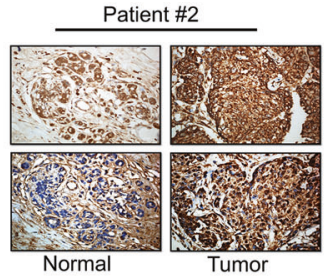

Patient \#5

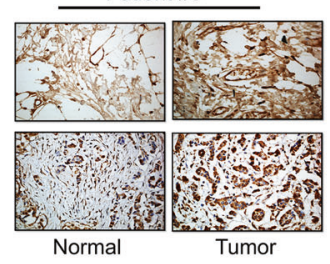

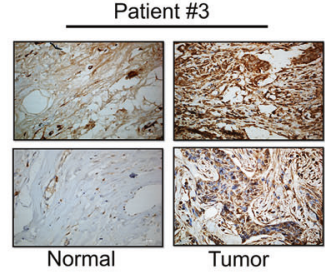

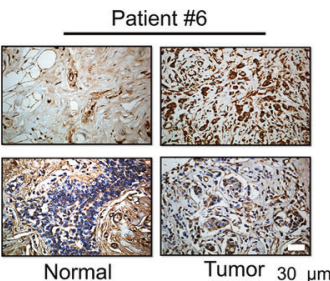

Fig. 7 USP3 overexpression in human breast cancer correlates with increased Cdc25A expression and poor prognosis. a Expression box plot showing significant differential expression of USP3 and Cdc25A in normal vs. tumor tissues using normal-tumor matched breast cancer patient RNAseq data from TCGA $(P$ value $<0.05)$. b Endogenous expression of USP 3 and Cdc25A in different cancerous and non-cancerous cell lines (HEK293 and hDPSc). c Kaplan-Meier survival curves. Breast cancer patients were divided into two groups

cell-derived DUBKO clones to screen functional DUBs for two main reasons. First, pooled batches of transfected cells targeting USPs generally contain a small fraction of cells modified with nuclease-induced genome editing, giving imprecise or false positive results. Second, some indel mutations in USP genes are expected to maintain the reading frame, which is critical because incomplete knockdown retains gene function and leads to the misinterpretation of results.

USP3 was recently implicated in the carcinogenesis and prognosis of gastric cancer [37]. A study has also shown that USP3 reverses double strand break-induced histone ubiquitination and delays $S$ phase progression [38]. Another study reported that a USP3-deleted mouse model had a shorter life span and increased cancer incidence compared with the controls [39]. In this study, we focused on the Cdc25A oncoprotein, which is associated with high-grade tumors and poor prognosis [40]. In conclusion, our study is the first attempt to perform genome-scale screening of functional USP family proteins using single cell-derived clones, and it identifies USP 3 as a stabilizer of Cdc25A phosphatase during cellcycle progression and tumorigenesis. We speculate that USP3 based on USP3 expression, and the overall survival data from the high and low groups were used for the Kaplan-Meier analysis $(P$ value $<$ 0.05). d Endogenous expression of USP 3 and Cdc25A in human breast cancer cell lines. e Representative immunohistochemical staining of endogenous USP3 and Cdc25A in human invasive ductal carcinoma of the breast (Tumor) and non-neoplastic tissue (Normal). Scale bar $=30 \mu \mathrm{m}$.

and Cdc25A could be critical targets for effective cancer therapeutics.

Acknowledgements We are grateful to all members of the SR and KSK labs, especially Naresh Poondla, Janardhan Karapurkar, and KiSang Jo, for their technical support and advice. We sincerely thank Dr Hyun-Hi Kim and Prof. Hansung Jung from Yonsei University for assisting in the IHC experiments. This research was supported by the Basic Science Research Program through the National Research Foundation of Korea (NRF), which is funded by the Ministry of Education (2018M3A9H3022412, 2017M3A9B3061830, and 2015H1D3A1036065).

\section{Compliance with ethical standards}

Conflict of interest The authors declare that they have no conflict of interest.

Publisher's note Springer Nature remains neutral with regard to jurisdictional claims in published maps and institutional affiliations.

\section{References}

1. Park MT, Lee SJ. Cell cycle and cancer. J Biochem Mol Biol. 2003;36:60-5. 
2. Vermeulen K, Van Bockstaele DR, Berneman ZN. The cell cycle: a review of regulation, deregulation and therapeutic targets in cancer. Cell Prolif. 2003;36:131-49.

3. Sur S, Agrawal DK. Phosphatases and kinases regulating CDC25 activity in the cell cycle: clinical implications of CDC25 overexpression and potential treatment strategies. Mol Cell Biochem. 2016;416:33-46.

4. Bernadette A, Bernard D. Cell cycle control by the CDC25 phosphatases. Anticancer Agents Med Chem. 2008;8:818-24.

5. Boutros R, Lobjois V, Ducommun B. CDC25 phosphatases in cancer cells: key players? Good targets? Nat Rev Cancer. 2007;7:495-507.

6. Xu X, Yamamoto H, Sakon M, Yasui M, Ngan CY, Fukunaga H, et al. Overexpression of CDC25A phosphatase is associated with hypergrowth activity and poor prognosis of human hepatocellular carcinomas. Clin Cancer Res. 2003;9:1764-72.

7. Donzelli M, Squatrito M, Ganoth D, Hershko A, Pagano M, Draetta GF. Dual mode of degradation of Cdc25 A phosphatase. EMBO J. 2002;21:4875-84.

8. Pereg Y, Liu BY, O’Rourke KM, Sagolla M, Dey A, Komuves L, et al. Ubiquitin hydrolase Dub3 promotes oncogenic transformation by stabilizing Cdc25A. Nat Cell Biol. 2010;12:400-6.

9. Ramakrishna S, Kwaku Dad A-B, Beloor J, Gopalappa R, Lee S$\mathrm{K}$, Kim $\mathrm{H}$. Gene disruption by cell-penetrating peptide-mediated delivery of Cas9 protein and guide RNA. Genome Res. 2014; 24:1020-7.

10. Kim HJ, Lee HJ, Kim H, Cho SW, Kim J-S. Targeted genome editing in human cells with zinc finger nucleases constructed via modular assembly. Genome Res. 2009;19:1279-88.

11. Kim S, Kim D, Cho SW, Kim J, Kim J-S. Highly efficient RNAguided genome editing in human cells via delivery of purified Cas9 ribonucleoproteins. Genome Res. 2014;24:1012-9.

12. Ramakrishna S, Cho SW, Kim S, Song M, Gopalappa R, Kim JS, et al. Surrogate reporter-based enrichment of cells containing RNA-guided Cas9 nuclease-induced mutations. Nat Commun. 2014;5:3378.

13. Latifi-Pupovci H, Kuçi Z, Wehner S, Bönig H, Lieberz R, Klingebiel $\mathrm{T}$, et al. In vitro migration and proliferation ("wound healing") potential of mesenchymal stromal cells generated from human CD271(+) bone marrow mononuclear cells. J Transl Med. 2015; $13: 315$.

14. Singh SK, Mishra MK, Eltoum I-EA, Bae S, Lillard JW, Singh R. CCR5/CCL5 axis interaction promotes migratory and invasiveness of pancreatic cancer cells. Sci Rep. 2018;8:1323.

15. Suresh B, Lee J, Hong SH, Kim KS, Ramakrishna S. The role of deubiquitinating enzymes in spermatogenesis. Cell Mol Life Sci. 2015;72:4711-20.

16. Kee Y, Huang TT. Role of deubiquitinating enzymes in DNA repair. Mol Cell Biol. 2016;36:524-44.

17. Chandrasekaran AP, Suresh B, Kim HH, Kim KS, Ramakrishna S. Concise review: fate determination of stem cells by deubiquitinating enzymes. Stem Cells. 2017;35:9-16.

18. Lim KH, Song MH, Baek KH. Decision for cell fate: deubiquitinating enzymes in cell cycle checkpoint. Cell Mol Life Sci. 2016;73:1439-55.

19. Liu Q, Wu Y, Qin Y, Hu J, Xie W, Qin FX-F, et al. Broad and diverse mechanisms used by deubiquitinase family members in regulating the type I interferon signaling pathway during antiviral responses. Sci Adv. 2018;4:eaar2824.

20. Biswas K, Philip S, Yadav A, Martin BK, Burkett S, Singh V, et al. BRE/BRCC45 regulates CDC25A stability by recruiting USP7 in response to DNA damage. Nat Commun. 2018;9:537.
21. Wu Y, Zhou L, Wang X, Lu J, Zhang R, Liang X, et al. A genomescale CRISPR-Cas9 screening method for protein stability reveals novel regulators of Cdc25A. Cell Discov. 2016;2:16014.

22. Busino L, Donzelli M, Chiesa M, Guardavaccaro D, Ganoth D, Valerio Dorrello N, et al. Degradation of Cdc25A by $\beta-\operatorname{TrCP}$ during S phase and in response to DNA damage. Nature. 2003; 426:87-91.

23. Mailand N, Falck J, Lukas C, Syljuåsen RG, Welcker M, Bartek J, et al. Rapid destruction of human Cdc25A in response to DNA damage. Science. 2000;288:1425-9.

24. Bernardi R, Liebermann DA, Hoffman B. Cdc25A stability is controlled by the ubiquitin-proteasome pathway during cell cycle progression and terminal differentiation. Oncogene. 2000;19:2447-54.

25. Young LM, Pagano M. Cdc25 phosphatases: differential regulation by ubiquitin-mediated proteolysis. Cell Cycle. 2010;9:4613-4.

26. Jin J, Shirogane T, Xu L, Nalepa G, Qin J, Elledge SJ, et al. SCFbeta-TRCP links Chk1 signaling to degradation of the Cdc25A protein phosphatase. Genes Dev. 2003;17:3062-74.

27. Emmerich $\mathrm{CH}$, Cohen P. Optimising methods for the preservation, capture and identification of ubiquitin chains and ubiquitylated proteins by immunoblotting. Biochem Biophys Res Commun. 2015;466:1-14.

28. Hjerpe R, Aillet F, Lopitz-Otsoa F, Lang V, England P, Rodriguez MS. Efficient protection and isolation of ubiquitylated proteins using tandem ubiquitin-binding entities. EMBO Rep. 2009;10:1250-8.

29. Hoffmann I. The role of $\mathrm{Cdc} 25$ phosphatases in cell cycle checkpoints. Protoplasma. 2000;211:8-11.

30. Shen T, Huang S. The role of Cdc25A in the regulation of cell proliferation and apoptosis. Anticancer Agents Med Chem. 2012; 12:631-9.

31. Timofeev O, Cizmecioglu O, Settele F, Kempf T, Hoffmann I. Cdc25 phosphatases are required for timely assembly of CDK1cyclin B at the G2/M transition. J Biol Chem. 2010;285:16978-90.

32. López-Contreras AJ, Fernandez-Capetillo O. The ATR barrier to replication-born DNA damage. DNA Repair. 2010;9:1249-55.

33. Cimprich KA, Cortez D. ATR: an essential regulator of genome integrity. Nat Rev Mol Cell Biol. 2008;9:616-27.

34. Ruiz S, Mayor-Ruiz C, Lafarga V, Murga M, Vega-Sendino M, Ortega S, et al. A Genome-wide CRISPR screen identifies CDC25A as a determinant of sensitivity to ATR inhibitors. Mol Cell. 2016;62:307-13.

35. Liu JC, Granieri L, Shrestha M, Wang D-Y, Vorobieva I, Rubie EA, et al. Identification of CDC25 as a common therapeutic target for triple-negative breast cancer. Cell Rep. 2018;23:112-26.

36. Cangi MG, Cukor B, Soung P, Signoretti S, Moreira G Jr., Ranashinge M, et al. Role of the Cdc25A phosphatase in human breast cancer. J Clin Investig. 2000;106:753-61.

37. Fang C-L, Lin C-C, Chen H-K, Hseu Y-C, Hung S-T, Sun D-P, et al. Ubiquitin-specific protease 3 overexpression promotes gastric carcinogenesis and is predictive of poor patient prognosis. Cancer Sci. 2018;109:3438-49.

38. Nicassio F, Corrado N, Vissers JHA, Areces LB, Bergink S, Marteijn JA, et al. Human USP3 is a chromatin modifier required for S phase progression and genome stability. Curr Biol. 2007; 17:1972-7.

39. Lancini C, van den Berk PCM, Vissers JHA, Gargiulo G, Song JY, Hulsman D, et al. Tight regulation of ubiquitin-mediated DNA damage response by USP3 preserves the functional integrity of hematopoietic stem cells. J Exp Med. 2014;211:1759-77.

40. Ray D, Kiyokawa H. CDC25A phosphatase: a rate-limiting oncogene that determines genomic stability. Cancer Res. 2008;68:1251-3. 